\title{
Local Lipschitzness of Reachability Maps for Hybrid Systems with Applications to Safety
}

\author{
Mohamed Maghenem \\ University of California, Santa Cruz \\ mmaghene@ucsc.edu
}

\begin{abstract}
Motivated by the safety problem, several definitions of reachability maps, for hybrid dynamical systems, are introduced. It is well established that, under certain conditions, the solutions to continuous-time systems depend continuously with respect to initial conditions. In such setting, the reachability maps considered in this paper are locally Lipschitz (in the Lipschitz sense for set-valued maps) when the right-hand side of the continuous-time system is locally Lipschitz. However, guaranteeing similar properties for reachability maps for hybrid systems is much more challenging. Examples of hybrid systems for which the reachability maps do not depend nicely with respect to their arguments, in the Lipschitz sense, are introduced. With such pathological cases properly identified, sufficient conditions involving the data defining a hybrid system assuring Lipschitzness of the reachability maps are formulated. As an application, the proposed conditions are shown to be useful to significantly improve an existing converse theorem for safety given in terms of barrier functions. Namely, for a class of safe hybrid systems, we show that safety is equivalent to the existence of a locally Lipschitz barrier function. Examples throughout the paper illustrate the results.
\end{abstract}

\section{CCS CONCEPTS}

- Theory of computation $\rightarrow$ Logic and verification; Modal and temporal logics; Linear logic; • Computer systems organization $\rightarrow$ Embedded and cyber-physical systems;

\section{KEYWORDS}

Reachable sets, Safety, Barrier functions, Hybrid systems

\section{ACM Reference Format:}

Mohamed Maghenem and Ricardo G. Sanfelice. 2020. Local Lipschitzness of Reachability Maps for Hybrid Systems with Applications to Safety. In 23rd ACM International Conference on Hybrid Systems: Computation and Control (HSCC '20), April 22-24, 2020, Sydney, NSW, Australia. ACM, New York, NY, USA, 11 pages. https://doi.org/10.1145/3365365.3382215

This research has been partially supported by the National Science Foundation under Grant no. ECS-1710621 and Grant no. CNS-1544396, by the Air Force Office of Scientific Research under Grant no. FA9550-16-1-0015, Grant no. FA9550-19-1-0053, and Grant no. FA9550-19-1-0169, and by CITRIS and the Banatao Institute at the University of California.

Permission to make digital or hard copies of all or part of this work for personal or classroom use is granted without fee provided that copies are not made or distributed for profit or commercial advantage and that copies bear this notice and the full citation on the first page. Copyrights for components of this work owned by others than ACM must be honored. Abstracting with credit is permitted. To copy otherwise, or republish, to post on servers or to redistribute to lists, requires prior specific permission and/or a fee. Request permissions from permissions@acm.org.

HSCC '20, April 22-24, 2020, Sydney, NSW, Australia

(C) 2020 Association for Computing Machinery.

ACM ISBN 978-1-4503-7018-9/20/04 _\$15.00

https://doi.org/10.1145/3365365.3382215

\author{
Ricardo G. Sanfelice \\ University of California, Santa Cruz \\ ricardo@ucsc.edu
}

\section{INTRODUCTION}

The reachable or attainable set for a dynamical system over a finite window of time can be seen as a set-valued map that maps each initial condition and time window to the set of points reached by the solutions from that initial condition during that time window $[1,2]$. Reachable sets are very useful, for example, in finite-horizon prediction and optimization problems [3]. One important property of reachable sets (when seen as set-valued maps) is their continuous dependence on their arguments - in particular, Lipschitz continuity. Indeed, for continuous-time systems such properties are nowadays considered as well-known facts, see, e.g., [4, 5]. The Lipschitz continuity of the reachability maps is key, for example, when analyzing continuous-time systems via their discretized version [6, 7]. Furthermore, continuity of reachability maps is shown in [8] to be very useful when studying the converse safety problem using barrier functions [9]. Indeed, the objective in the converse safety problem is to show the existence of a barrier function when the system is safe; namely, when the solutions starting from a given initial set never reach a given unsafe set $[10,11]$. Such a barrier certificate is show in [8] to inherit the regularity properties of the reachable sets.

In the context of hybrid systems modeled according to the framework in [12], there are several possible definitions of reachable sets, see [12, Section 6.3.2], [13], and [8]. This flexibility in the formulation of reachable sets is due to the solutions being defined on a hybrid time domain indexing both the duration of the continuoustime evolution (the flow) as well as the amount of jumps. In such a context, and to the best of our knowledge, the continuous dependence of the reachability maps on there arguments is not fully documented in the literature. Moreover, extending the existing results for continuous-time systems is not straightforward. Indeed, in the general case of hybrid systems, even when the dynamics are defined by single-valued smooth maps, reachability maps can fail to be continuous with respect to their arguments. Such a pathology is mainly due to the evolution of the solutions to hybrid systems being constrained by the so-called flow and jump sets. This fact is illustrated in [8, Example 4.4] for a canonical hybrid system, the bouncing ball. This challenge motivates the work in this paper about identifying the tightest possible regularity on the data of the hybrid system that allow the best possible regularity properties for the considered reachability maps.

In this paper, we first reconsider the two reachability maps, denoted $R$ and $\widehat{R}$, introduced in [8]. The map $R$ provides the set of points reached by the solutions starting from a given initial condition $x_{o}$ during a given flow time window and without exceeding a given number of jumps $J$. The map $\widehat{R}$ is a prolongation of $R$ using the solutions to the system. The map $\widehat{R}$ includes not only the elements reached without exceeding the given flow time window and the given number of jumps $J$, but also the elements reached while exceeding the given flow time window until $J$ jumps are achieved, if possible (without exceeding $J$ jumps). In the particular case of 
continuous-time systems, the maps $R$ and $\widehat{R}$ coincide and reduce to the one in [1]. In [8], semicontinuity and boundedness properties of both $R$ and $\widehat{R}$ are analyzed in the context of well-posed hybrid systems. Here, we also introduce the maps $R^{b}$ and $\widehat{R}^{b}$ that include the elements in $R$ and $\widehat{R}$, respectively, that are the last to reach by each maximal solution starting from $x_{o}$. For continuous-time systems, the maps $R^{b}$ and $\widehat{R}^{b}$ coincide and reduce to the reachability map studied in $[4,6,14]$. Using these definitions, this paper makes the following contributions:

- In the first and main part of this paper, we analyze the local Lipschitzness of the proposed reachability maps after handling the pathologies preventing such regularity via a set of conditions on the data of the hybrid system.

- In the second part of this paper, the aforementioned study is used to improve the converse safety result in [8]. For this purpose, we use the fact that the barrier function used in [8] inherits the regularity properties of the reachability map $\widehat{R}$. As a result, for the considered class of hybrid systems, we show that safety is equivalent to the existence of a locally Lipschitz barrier function satisfying sufficient infinitesimal conditions for safety.

The rest of the paper is organized as follows. Preliminaries are in Section 2. The considered reachability maps are in Section 3. The pathologies preventing the Lipschitz continuity of the considered maps are in Section 4. The main results are in Section 5. Finally, the application to the converse safety problem is in Section 6 .

Due to space constraints, the proofs are omitted and will be published elsewhere.

Notation. Let $\mathbb{R}_{\geq 0}:=[0, \infty)$ and $\mathbb{N}:=\{0,1, \ldots, \infty\}$. For $x$, $y \in \mathbb{R}^{n}, x^{\top}$ denotes the transpose of $x,|x|$ the Euclidean norm of $x,|x|_{K}:=\inf _{y \in K}|x-y|$ defines the distance between $x$ and the nonempty set $K$, and $\langle x, y\rangle=x^{\top} y$ denotes the scalar product between $x$ and $y$. For a set $K \subset \mathbb{R}^{n}$, we use $\operatorname{int}(K)$ to denote its interior, $\partial K$ to denote its boundary, $\operatorname{cl}(K)$ to denote its closure, and $U(K)$ to denote any open neighborhood of $K$. For a set $O \subset \mathbb{R}^{n}, K \backslash O$ denotes the subset of elements of $K$ that are not in $O$. By $\mathbb{B}$, we denote the closed unit ball in $\mathbb{R}^{n}$ centered at the origin. Finally, $F: \mathbb{R}^{m} \rightrightarrows \mathbb{R}^{n}$ denotes a set-valued map associating each element $x \in \mathbb{R}^{m}$ into a set $F(x) \subset \mathbb{R}^{n}$.

\section{PRELIMINARIES}

\subsection{Set-Valued and Nonsmooth Analysis}

We start this section by recalling the following semicontinuity and boundedness notions $[15,16]$.

Definition 1 (Semicontinuous SET-VAlued maPs). Consider $a$ set-valued map $F: \mathbb{R}^{m} \rightrightarrows \mathbb{R}^{n}$.

- The map $F$ is said to be outer semicontinuous at $x \in \mathbb{R}^{m}$ if, for all $\left\{x_{i}\right\}_{i=0}^{\infty} \subset \mathbb{R}^{m}$ and for all $\left\{y_{i}\right\}_{i=0}^{\infty} \subset \mathbb{R}^{n}$ with $x_{i} \rightarrow x$, $y_{i} \in F\left(x_{i}\right)$, and $y_{i} \rightarrow y \in \mathbb{R}^{n}$, we have $y \in F(x)$; see [12, Definition 5.9].

- The map F is said to be lower semicontinuous (or, equivalently, inner semicontinuous) at $x \in \mathbb{R}^{m}$ if, for each $\epsilon>0$ and for each $y_{x} \in F(x)$, there exists $U(x)$ such that, for each $z \in U(x)$, there exists $y_{z} \in F(z)$ such that $\left|y_{z}-y_{x}\right| \leq \epsilon$; see [17, Proposition 2.1].

- The map $F$ is said to be upper semicontinuous at $x \in \mathbb{R}^{m}$ if, for each $\epsilon>0$, there exists $U(x)$ such that, for each $y \in U(x)$, $F(y) \subset F(x)+\epsilon \mathbb{B}$; see [15, Definition 1.4.1].
- The map $F$ is said to be continuous at $x \in \mathbb{R}^{m}$ if it is both upper and lower semicontinuous at $x$.

Furthermore, the map $F$ is said to be upper, lower, outer semicontinuous, or continuous if it is upper, lower, outer semicontinuous, or continuous for all $x \in \mathbb{R}^{m}$, respectively.

Definition 2 (Semicontinuous single-valued maps). Consider a scalar function $B: \mathbb{R}^{m} \rightarrow \mathbb{R}$.

- The scalar function $B$ is said to be lower semicontinuous at $x \in \mathbb{R}^{m}$ if, for every sequence $\left\{x_{i}\right\}_{i=0}^{\infty} \subset \mathbb{R}^{m}$ such that $\lim _{i \rightarrow \infty} x_{i}=x$, we have $\liminf _{i \rightarrow \infty} B\left(x_{i}\right) \geq B(x)$.

- The scalar function $B$ is said to be upper semicontinuous at $x \in \mathbb{R}^{m}$ if, for every sequence $\left\{x_{i}\right\}_{i=0}^{\infty} \subset \mathbb{R}^{m}$ such that $\lim _{i \rightarrow \infty} x_{i}=x$, we have $\limsup _{i \rightarrow \infty} B\left(x_{i}\right) \leq B(x)$.

- The scalar function $B$ is said to be continuous at $x \in \mathbb{R}^{m}$ if it is both upper and lower semicontinuous at $x$.

Furthermore, $B$ is said to be upper, lower semicontinuous, or continuous if it is upper, lower semicontinuous, or continuous for all $x \in \mathbb{R}^{m}$, respectively.

Definition 3 (LocAlly Lipschitz MAPS). The set-valued map $F: M\left(\subset \mathbb{R}^{m}\right) \rightrightarrows \mathbb{R}^{n}$ is said to be locally Lipschitz around $x \in M$ if there exist $U(x)$ and $k>0$ such that, for all $\left(x_{1}, x_{2}\right) \in(U(x) \cap M) \times$ $(U(x) \cap M)$,

$$
F\left(x_{1}\right) \subset F\left(x_{2}\right)+k\left|x_{1}-x_{2}\right| \mathbb{B} .
$$

Furthermore, the set-valued map $F: M \rightrightarrows \mathbb{R}^{n}$ is said to be locally Lipschitz on $M^{\prime} \subset M$ if so is the map $F: M^{\prime} \rightrightarrows \mathbb{R}^{n}$ around each $x \in M^{\prime}$. by

The proximal normal cone of a set $K \subset \mathbb{R}^{n}$ at $x \in \operatorname{cl}(K)$ is given

$$
N_{K}^{P}(x):=\left\{\zeta \in \mathbb{R}^{n}: \exists t>0 \text { such that }|x+t \zeta|_{K}=t|\zeta|\right\} .
$$

Furthermore, according to the same reference, the proximal subdifferential of a lower semicontinuous function $B: \mathbb{R}^{n} \rightarrow \mathbb{R}$, denoted by $\partial_{P} B$, evaluated at $x \in \mathbb{R}^{n}$ is a subset of the normal proximal cone $N_{\text {epi } B}^{P}((x, B(x)))$, where epi $B$ is the epigraph of $B$; namely,

$$
\text { ерi } B:=\left\{(x, r) \in \mathbb{R}^{n} \times \mathbb{R}: r \geq B(x)\right\} .
$$

DEFINITION 4. The proximal subdifferential of a lower semicontinuous function $B: \mathbb{R}^{n} \rightarrow \mathbb{R}$ is the set-valued map $\partial_{P} B: \mathbb{R}^{n} \rightrightarrows \mathbb{R}^{n}$ defined for all $x \in \mathbb{R}^{n}$ as

$$
\partial_{P} B(x):=\left\{\zeta \in \mathbb{R}^{n}:\left[\zeta^{\top}-1\right]^{\top} \in N_{\text {epiB }}^{P}(x, B(x))\right\} .
$$

Moreover, each vector $\zeta \in \partial_{P} B(x)$ is said to be a proximal subgradient of $B$ at $x$.

\subsection{Hybrid Systems}

Following the framework proposed in [12], a hybrid dynamical system $\mathcal{H}=(C, F, D, G)$ is modeled as

$$
\mathcal{H}:\left\{\begin{array}{lr}
x \in C & \dot{x} \in F(x) \\
x \in D & x^{+} \in G(x),
\end{array}\right.
$$

with the state variable $x \in \mathbb{R}^{n}$, the flow set $C \subset \mathbb{R}^{n}$, the jump set $D \subset \mathbb{R}^{n}$, and the flow and the jump set-valued maps, respectively, $F: \mathbb{R}^{n} \rightrightarrows \mathbb{R}^{n}$ and $G: \mathbb{R}^{n} \rightrightarrows \mathbb{R}^{n}$.

A hybrid arc $\phi$ is defined on a hybrid time domain denoted $\operatorname{dom} \phi \subset \mathbb{R}_{\geq 0} \times \mathbb{N}$. The hybrid arc $\phi$ is parametrized by an ordinary time variable $t \in \mathbb{R}_{\geq 0}$ and a discrete jump variable $j \in \mathbb{N}$. Its domain of definition $\operatorname{dom} \phi$ is such that for each $(T, J) \in \operatorname{dom} \phi$, 


$$
\begin{aligned}
& \operatorname{dom} \phi \cap([0, T] \times\{0,1, \ldots, J\})=\cup_{j=0}^{J-1}\left(\left[t_{j}, t_{j+1}\right] \times\{j\}\right) \text { for a se- } \\
& \text { quence }\left\{t_{j}\right\}_{j=0}^{J+1} \text {, such that } t_{j+1} \geq t_{j} \text { and } t_{0}=0
\end{aligned}
$$

Definition 5 (Concept of Solution to $\mathcal{H}$ ). A hybrid arc $\phi$ : $\operatorname{dom} \phi \rightarrow \mathbb{R}^{n}$ is a solution to $\mathcal{H}$ if

(So) $\phi(0,0) \in \operatorname{cl}(C) \cup D$;

(S1) for all $j \in \mathbb{N}$ such that $I^{j}:=\{t:(t, j) \in \operatorname{dom} \phi\}$ has nonempty interior, $t \mapsto \phi(t, j)$ is locally absolutely continuous and

$$
\begin{aligned}
& \phi(t, j) \in C \quad \text { for all } \quad t \in \operatorname{int}\left(I^{j}\right) \text {, } \\
& \dot{\phi}(t, j) \in F(\phi(t, j)) \quad \text { for almost all } t \in I^{j} \text {; }
\end{aligned}
$$

(S2) for all $(t, j) \in \operatorname{dom} \phi$ such that $(t, j+1) \in \operatorname{dom} \phi$,

$$
\phi(t, j) \in D, \quad \phi(t, j+1) \in G(\phi(t, j)) .
$$

A solution $\phi$ to $\mathcal{H}$ is said to be maximal if there is no solution $\psi$ to $\mathcal{H}$ such that $\phi(t, j)=\psi(t, j)$ for all $(t, j) \in \operatorname{dom} \phi$ with dom $\phi$ a proper subset of dom $\psi$. It is said to be trivial if $\operatorname{dom} \phi$ contains only one element. The system $\mathcal{H}$ is said to be complete if the domain of each maximal solution is unbounded. It is said to be pre-complete if the domain of each maximal solution is closed. Finally, we use $\hat{\mathcal{S}}_{\mathcal{H}}\left(x_{o}\right)$ to denote the set of solutions to $\mathcal{H}$ starting from $x_{o} \in \operatorname{cl}(C) \cup D$. Finally, we use reach $\left(x_{o}\right)$ to denote the set generated by the maximal solutions starting from $x_{o} \in \operatorname{cl}(C)$; namely,

$$
\operatorname{reach}\left(x_{o}\right):=\left\{\phi(t, j):(t, j) \in \operatorname{dom} \phi, \phi \in \hat{\mathcal{S}}_{\mathcal{H}}\left(x_{o}\right)\right\} .
$$

A hybrid $\operatorname{arc} \phi$ is a backward solution to $\mathcal{H}$ if there exists a solution $\psi$ to the hybrid system $\mathcal{H}^{-}$such that $\operatorname{dom} \phi=-\operatorname{dom} \psi$ and $\psi(t, j)=\phi(-t,-j)$ for all $(t, j) \in \operatorname{dom} \psi$, where

$$
\mathcal{H}^{-}:\left\{\begin{array}{cc}
x \in C & \dot{x} \in-F(x) \\
x \in G(D) & x^{+} \in G_{D}^{-1}(x)
\end{array}\right.
$$

and $G_{D}^{-1}: G(D) \rightrightarrows \mathbb{R}^{n}$ is the reciprocal map of the jump map $G$ restricted to the set $D$; namely,

$$
G_{D}^{-1}(y):=\{x \in D: y \in G(x)\} .
$$
as:

Furthermore, for each $x \in \operatorname{cl}(C) \cup D$, we introduce the set $\hat{\mathcal{S}}_{\mathcal{H}}^{-}(x)$

- The set of backward solutions to $\mathcal{H}$ starting from $x$ if $x \in$ $\operatorname{cl}(C) \cup(G(D) \cap D)$.

- The trivial hybrid arc starting from $x$, otherwise.

According to [12], a hybrid inclusion $\mathcal{H}=(C, F, D, G)$ is said to satisfy the hybrid basic conditions if the following conditions are satisfied.

(A1) The sets $C$ and $D$ are closed.

(A2) The flow map $F: \mathbb{R}^{n} \rightrightarrows \mathbb{R}^{n}$ is outer semicontinuous and locally bounded relative to $C$, and $F(x)$ is nonempty and convex for all $x \in C$.

(A3) The jump map $G: \mathbb{R}^{n} \rightrightarrows \mathbb{R}^{n}$ is outer semicontinuous relative to $D$ and $G(x)$ is nonempty for all $x \in D$.

(A4) The jump map $G: \mathbb{R}^{n} \rightrightarrows \mathbb{R}^{n}$ is locally bounded relative to $D$.

REMARK 1. The hybrid basic conditions (A1)-(A4) are shown in [12] to guarantee very useful structural properties for the set of solutions to $\mathcal{H}$. Due to this, $\mathcal{H}$ satisfying (A1)-(A4) is said to be well posed.

\subsection{Minimal-Time Functions for Constrained Differential Inclusions}

In this section, we recall from [18] the definition of the minimaltime function with respect to a closed the set $K \subset C$ for the continuous dynamics of $\mathcal{H}$ given by

$$
\mathcal{H}_{f}: \quad \dot{x} \in F(x) \quad x \in C .
$$

Similar to the notion of solutions to $\mathcal{H}$, a solution $\phi$ to $\mathcal{H}_{f}$ is defined as a solution to $\mathcal{H}$ that never jumps and $\operatorname{dom} \phi \subset \mathbb{R}_{\geq 0}$.

Definition 6. The minimal-time function $t_{K}^{\text {min }}: \operatorname{cl}(C) \rightarrow \mathbb{R}_{\geq 0}$ with respect to a closed the set $K \subset \mathrm{cl}(C)$ and for a constrained system $\mathcal{H}_{f}=(C, F)$ is given by

$$
t_{K}^{\min }\left(x_{o}\right):=\left\{\begin{array}{cc}
+\infty & \text { ifreach }\left(x_{o}\right) \cap K=\emptyset \\
0 & \text { if } x_{o} \in K \\
\min _{\substack{\phi(t) \in K \\
t \in \operatorname{dom} \phi \\
\phi \in \hat{\mathcal{S}}_{\mathcal{H}_{f}}\left(x_{o}\right)}} t & \text { otherwise. }
\end{array}\right.
$$

The minimal-time function $t_{K}^{\text {min }}$ in Definition 6 provides the first time that a solution to $\mathcal{H}_{f}$ starting from $x_{o} \in \operatorname{cl}(C)$ reaches the set $K$. If all the solutions starting from $x_{o}$ never reach the set $K$, the minimal-time function is set to infinity. In [18, Theorem 1], we proposed necessary and sufficient conditions such that the minimal-time function $t_{K}^{\text {min }}$ introduced in Definition 6 is locally Lipschitz on the set $S_{K}^{\min }$ defined by

$$
S_{K}^{\text {min }}:=\left\{x \in \operatorname{cl}(C): t_{K}^{\min }(x)<+\infty\right\} .
$$

The proposed conditions in [18] are infinitesimal; i.e., they involve only the sets $K$ and $C$, and the map $F$.

Furthermore, given a closed set $K \subset \operatorname{cl}(C)$, we define the function $t_{K}: \operatorname{cl}(C) \rightarrow \mathbb{R}_{\geq 0}$ as

$t_{K}\left(x_{o}\right):=\left\{\begin{array}{ccc}t_{K}^{\min }\left(x_{o}\right) & \text { if } x_{o} \notin K \text { or } \hat{\mathcal{S}}_{\mathcal{H}_{f}}\left(x_{o}\right) \text { is trivial, } \\ \inf _{\phi \in \hat{\mathcal{S}}_{\mathcal{H}_{f}}\left(x_{o}\right)} \liminf _{t \rightarrow 0^{+}} t_{K}^{\min }(\phi(t)) & \text { otherwise. }\end{array}\right.$

The only difference between $t_{K}$ and $t_{K}^{m i n}$ is that, when $x_{o} \in K$ and the maximal solutions starting from $x_{o}$ immediately leave the set $K, t_{K}\left(x_{o}\right)$ provides the next time, after the initial time, at which a maximal solution from $x_{o}$ reaches the set $K$. The latter is captured by the "otherwise" piece in (14).

Lipschitz continuity of the function $t_{K}$ can be deduced, in some cases, from to Lipschitz continuity of the minimal-time function $t_{K_{a}}^{m i n}$ with respect to a subset $K_{a} \subset K$ as shown in [18].

The following example illustrates this point.

EXAmple 1. [Bouncing ball] The continuous dynamics of the bouncing ball hybrid model is given by $\mathcal{H}_{f}:=(C, F)$, where $F(x):=$ $\left[\begin{array}{ll}x_{2} & -\gamma\end{array}\right]^{\top}$ for each $C:=\left\{x \in \mathbb{R}^{2}: x_{1} \geq 0\right\}$, and the constant $\gamma>0$ is the gravity acceleration. Furthermore, we consider the (jump) set

$$
D:=\left\{x \in \mathbb{R}^{2}: x_{1}=0, x_{2} \leq 0\right\} \subset \partial C .
$$

Next, we consider the sets $\left(K, K_{a}\right)=(\partial C, D)$ and we show that $t_{\partial C}(x)=t_{D}^{\min }(x)$ for each $x \in C$. Indeed, it is easy to see that the 
solutions to $\mathcal{H}_{f}$ starting from $D$ are trivial. Furthermore, the set $D$ is closed and is a subset of $\partial C$. Hence, for all $x \in D, t_{\partial C}(x)=0$. Furthermore, for all $x \in \partial C \backslash D, t_{\partial C}(x)>0$ since the solutions starting from $\partial C \backslash D$ flow immediately in $\operatorname{int}(C)$ and they remain in $\operatorname{int}(C)$ until they reach the set $D$.

The Lipschitz continuity of $t_{K}$ on the set

$$
S_{K}:=\left\{x \in \operatorname{cl}(C): t_{K}(x)<\infty\right\}
$$

will play a key role when analyzing the Lipschitz continuity of the reachablity maps for hybrid systems.

\subsection{Monotonicity Along Solutions to Constrained Differential Inclusions}

In this section, we recall from [19] necessary and sufficient infinitesimal conditions such that a lower semicontinuous function $B: \mathbb{R}^{n} \rightarrow \mathbb{R}$ satisfies the following monotonicity property:

$(\star)$ The function $B$ is nonincreasing along the solutions to $\mathcal{H}_{f}=$ $(C, F)$; namely, for every solution $t \mapsto \phi(t)$, the map $t \mapsto$ $B(\phi(t))$ is nonincreasing. Equivalently, $B\left(\phi\left(t_{1}\right)\right) \leq B\left(\phi\left(t_{2}\right)\right)$ for all $\left(t_{1}, t_{2}\right) \in \operatorname{dom} \phi \times \operatorname{dom} \phi$ with $t_{1} \geq t_{2}$.

To do so, we consider the following assumptions:

(a1) $F: \mathbb{R}^{n} \rightrightarrows \mathbb{R}^{n}$ is locally Lipschitz.

(a2) $F(x)$ is convex and closed for all $x \in C$.

(a3) For every nontrivial solution $\phi$ starting from $x_{o} \in \partial C$, there exists $\epsilon>0$ such that $\phi\left((0, \epsilon], x_{o}\right) \subset \operatorname{int}(C)$.

(a4) $B$ is continuous on $\partial C \cap \widetilde{C}$, where

$$
\widetilde{C}:=\left\{x_{o} \in \operatorname{cl}(C): \exists \phi \in \hat{\mathcal{S}}_{\mathcal{H}}\left(x_{o}\right), \operatorname{dom} \phi \neq\{0\}\right\} .
$$

Furthermore, we consider the following infinitesimal condition:

$$
\langle\zeta, \eta\rangle \leq 0 \quad \forall \zeta \in \partial_{P} B(x), \forall \eta \in F(x), \forall x \in \operatorname{int}(C) .
$$

Lemma 2.1. [19, Corollary 4.13] Consider a constrained system $\mathcal{H}_{f}=(C, F)$ such that (a1)-(a2) hold and let $B: \mathbb{R}^{n} \rightarrow \mathbb{R}$ be a lower semicontinuous function. Then,

- $(\star) \Rightarrow(18)$.

- When $\widetilde{C}$ is open or when (a3)-(a4) hold, $(\star) \Leftrightarrow(18)$.

\section{REACHABILITY MAPS FOR HYBRID SYSTEMS}

In this section, we introduce the reachability maps studied in this paper. In the case of hybrid systems, different definitions of reachability maps are available in the literature [12, Section 6.3.2] In this paper, the maps we propose are those we find the most helpful to study the converse safety problem in Section 6 in the sense that they allow useful continuity properties with respect to their arguments.

Given $x_{o} \in \operatorname{cl}(C) \cup D, T \in \mathbb{R}_{\geq 0}, J \in \mathbb{N}$, and $\mathcal{T}(T, J):=[0, T] \times$ $\{0,1, \ldots, J\}$. Similar to [8], we define the reachability map $R: \mathbb{R}_{\geq 0} \times$ $\mathbb{N} \times(\operatorname{cl}(C) \cup D) \rightrightarrows \operatorname{cl}(C) \cup D \cup G(D)$ as

$$
R\left(T, J, x_{o}\right):=\left\{\phi(t, j): \phi \in \hat{\mathcal{S}}_{\mathcal{H}}\left(x_{o}\right),(t, j) \in \operatorname{dom} \phi \cap \mathcal{T}(T, J)\right\} .
$$

The map $R\left(T, J, x_{o}\right)$ provides the set reached by the solutions starting from the initial condition $x_{o}$ during the interval of flow $[0, T]$ and without exceeding $J$ number of jump.

Furthermore, given $x_{o} \in \operatorname{cl}(C) \cup D, T \in \mathbb{R}_{\geq 0}, J \in \mathbb{N}$, and $\mathcal{T}(T, J):=[0, T] \times\{0,1, \ldots, J\}$, we define the reachability map

$$
\begin{aligned}
R^{b}: & \mathbb{R}_{\geq 0} \times \mathbb{N} \times(\operatorname{cl}(C) \cup D) \rightrightarrows \operatorname{cl}(C) \cup D \cup G(D) \text { as } \\
R^{b}\left(T, J, x_{o}\right) & :=\left\{\phi(t, j): \phi \in \hat{\mathcal{S}}_{\mathcal{H}}\left(x_{o}\right),(t, j) \in \operatorname{dom} \phi \cap \mathcal{T}(T, J),\right. \\
\nexists\left(t^{\prime}, j^{\prime}\right) & \left.\in \operatorname{dom} \phi \cap \mathcal{T}(T, J) \text { s.t. } t^{\prime}+j^{\prime}>t+j\right\}
\end{aligned}
$$

The map $R^{b}\left(T, J, x_{o}\right)$ contains the elements of $R\left(T, J, x_{o}\right)$ that are the last to reach by the maximal solution starting from $x_{o}$.

We also recall the reachability map introduced in [8]. Given $x_{o} \in \operatorname{cl}(C) \cup D, T \in \mathbb{R}_{\geq 0}$, and $J \in \mathbb{N}$, the map $\widehat{R}: \mathbb{R}_{\geq 0} \times \mathbb{N} \times(\operatorname{cl}(C) \cup$ $D) \rightrightarrows \mathrm{cl}(C) \cup D$ is defined as

$$
\widehat{R}\left(T, J, x_{o}\right):=\left\{\phi(t, j): \phi \in \hat{\mathcal{S}}_{\mathcal{H}}\left(x_{o}\right),(t, j) \in \operatorname{dom} \phi \cap \mathcal{T}_{\phi}(T, J)\right\},
$$

where

$$
\begin{gathered}
\mathcal{T}_{\phi}(T, J):=\left[0, T+\delta_{\phi}(T, J)\right] \times\{0,1, \ldots, J\} \\
\delta_{\phi}(T, J):= \\
\left\{\begin{array}{cc}
\min \left\{\delta \geq 0:\left(T+\delta, J_{\phi}(J)\right) \in \operatorname{dom} \phi\right\} & \text { if } I^{J_{\phi}(J)} \cap[0, T]=\emptyset \\
0 & \text { otherwise },
\end{array}\right.
\end{gathered}
$$

$$
J_{\phi}(J):=\max \{j \leq J: \exists t \geq 0:(t, j) \in \operatorname{dom} \phi\},
$$

and

$$
I^{J_{\phi}(J)}:=\left\{t \in \mathbb{R}_{\geq 0}:\left(t, J_{\phi}(J)\right) \in \operatorname{dom} \phi\right\} .
$$

The reachability map $\widehat{R}\left(T, J, x_{o}\right)$ includes not only the elements reached by the maximal solutions starting from $x_{o}$ over the hybrid window $\mathcal{T}(T, J)$, but also the elements reached by each maximal solution $\phi$ starting from $x_{o}$ after time $T$ until the jump $J_{\phi}(J) \leq J$ happens, if the latter happens after time $T$. The value of $J_{\phi}(J)$ is the last jump that the maximal solution $\phi$ achieves on the hybrid interval $\mathbb{R}_{\geq 0} \times\{0,1, \ldots, J\}$.

Finally, given $x_{o} \in \operatorname{cl}(C) \cup D, T \in \mathbb{R}_{\geq 0}$, and $J \in \mathbb{N}$, the reachability $\operatorname{map} \widehat{R}^{b}: \mathbb{R}_{\geq 0} \times \mathbb{N} \times(\operatorname{cl}(C) \cup D) \rightrightarrows \operatorname{cl}(C) \cup D \cup G(D)$ is defined as

$$
\begin{gathered}
\widehat{R}^{b}\left(T, J, x_{o}\right):=\left\{\phi(t, j): \phi \in \hat{\mathcal{S}}_{\mathcal{H}}\left(x_{o}\right),(t, j) \in \operatorname{dom} \phi \cap \mathcal{T}_{\phi}(T, J),\right. \\
\left.\nexists\left(T^{\prime}, J^{\prime}\right) \in \mathcal{T}_{\phi}(T, J) \cap \operatorname{dom} \phi \text { s.t. } T^{\prime}+J^{\prime}>T+J\right\} .
\end{gathered}
$$

The map $\widehat{R}^{b}\left(T, J, x_{o}\right)$ contains the elements of $\widehat{R}\left(T, J, x_{o}\right)$ that are the last to reach by the maximal solutions starting from $x_{o}$.

REMARK 2. When there is a unique solution $\phi$ to $\mathcal{H}$ starting from $x_{o} \in \operatorname{cl}(C) \cup D$, we conclude that, for all $(T, J) \in \mathbb{R}_{\geq 0} \times \mathbb{N}$,

and

$$
\widehat{R}\left(T, J, x_{o}\right)=R\left(T+\delta_{\phi}(T, J), J, x_{o}\right)
$$

$$
\widehat{R}^{b}\left(T, J, x_{o}\right)=R^{b}\left(T+\delta_{\phi}(T, J), J, x_{o}\right)
$$

The different reachability maps introduced above are illustrated in the following example:

EXAMPLE 2. Consider the hybrid dynamical system modeling the bouncing ball with the following data:

$$
\begin{gathered}
F(x):=\left[\begin{array}{ll}
x_{2} & -\gamma
\end{array}\right]^{\top} \quad \forall x \in C:=\left\{x \in \mathbb{R}^{2}: x_{1} \geq 0\right\}, \\
G(x):=\left[\begin{array}{ll}
0 & -\lambda x_{2}
\end{array}\right]^{\top} \quad \forall x \in D:=\left\{x \in \mathbb{R}^{2}: x_{1}=0, x_{2} \leq 0\right\} .
\end{gathered}
$$

The constants $\gamma>0$ and $\lambda \in[0,1]$ are the gravity acceleration and the restitution coefficient, respectively. Let $x_{o}:=\left[\begin{array}{ll}x_{o 1} & x_{o 2}\end{array}\right]^{\top} \in$ 
$\operatorname{int}(C) \backslash D$ (i.e., $x_{o 1}>0$ ) and let $\phi$ be the (unique) maximal solution starting from $x_{o}$. Furthermore, let $T_{o} \geq 0$ be the time at which the solution $\phi$ achieves the first jump, which is given by $T_{o}=\frac{x_{o 2}+\sqrt{x_{o 2}^{2}+2 \gamma x_{o 1}}}{\gamma}$. Now, for each $T \in\left(0, T_{o}\right)$, the reachability map in (19) results in $R\left(T, 1, x_{o}\right)=R\left(T, 0, x_{o}\right)=\phi([0, T], 0)=$ $\cup_{s=0}^{T}\left\{\left[\begin{array}{ll}-\frac{1}{2} \gamma s^{2}+x_{o 2} s+x_{o 1} & -\gamma s+x_{o 2}\end{array}\right]^{\top}\right\}$ and the the reachability map in (20) results in $R^{b}\left(T, 1, x_{0}\right)=R^{b}\left(T, 0, x_{o}\right)=\phi(T, 0)=$ $\left[\begin{array}{ll}-\frac{1}{2} \gamma T^{2}+x_{o 2} T+x_{o 1} & -\gamma T+x_{o 2}\end{array}\right]^{\top}$. Now, using the definitions in (21) and (26), we compute $\widehat{R}\left(T, 1, x_{o}\right)$ and $\widehat{R}^{b}\left(T, 1, x_{o}\right)$ for the same initial condition $x_{o}$ and for $T \in\left(0, T_{o}\right)$. Using (23), we conclude that $\delta_{\phi}(T, 1)=T_{o}-T$. Hence, we obtain

$$
\begin{aligned}
\widehat{R}\left(T, 1, x_{o}\right)= & R\left(T_{o}, 1, x_{o}\right)=\phi\left(\left[0, T_{o}\right], 0\right) \bigcup\left\{\phi\left(T_{o}, 1\right)\right\} \\
= & \phi\left(\left[0, T_{o}\right], 0\right) \cup\left\{G\left(\phi\left(T_{o}, 0\right)\right)\right\} \\
= & \bigcup_{s=0}^{T_{o}}\left\{\left[\begin{array}{ll}
-\gamma s^{2} / 2+x_{o 2} s+x_{o 1} & -\gamma s+x_{o 2}
\end{array}\right]^{\top}\right\} \\
& \cup\left\{\left[\begin{array}{ll}
0 & \lambda\left(x_{o 2}^{2}+2 \gamma x_{o 1}\right)^{\frac{1}{2}}
\end{array}\right]^{\top}\right\}
\end{aligned}
$$

and

$$
\begin{aligned}
\widehat{R}^{b}\left(T, 1, x_{o}\right) & =R^{b}\left(T_{o}, 1, x_{o}\right)=\phi\left(T_{o}, 1\right)=G\left(\phi\left(T_{o}, 0\right)\right) \\
& =\left[\begin{array}{ll}
0 & \lambda\left(x_{o 2}^{2}+2 \gamma x_{o 1}\right)^{\frac{1}{2}}
\end{array}\right]^{\top} .
\end{aligned}
$$

Finally, we notice that, for all $T \geq T_{o}$,

$$
\widehat{R}\left(T, 1, x_{o}\right)=R\left(T, 1, x_{o}\right) \quad \text { and } \quad \widehat{R}^{b}\left(T, 1, x_{o}\right)=R^{b}\left(T, 1, x_{o}\right) .
$$

Remark 3. When $\mathcal{H}=(C, F, \emptyset, \star)$, namely, $\mathcal{H}$ is a constrained differential inclusion, we conclude that, for all $(T, x) \in \mathbb{R}_{\geq 0} \times \operatorname{cl}(C)$,

$$
R(T, J, x)=\widehat{R}(T, J, x)=: \tilde{R}(T, x) \quad \forall J \in \mathbb{N}
$$

and

$$
R^{b}(T, J, x)=\widehat{R}^{b}(T, J, x)=: \tilde{R}^{b}(T, x) \quad \forall J \in \mathbb{N} .
$$

REMARK 4. In [8], the continuity properties for the maps $R$ and $\widehat{R}$ are analyzed and it is shown that, when $\mathcal{H}$ satisfies the hybrid basic conditions (A1)-(A4), the map $R$ is outer semicontinuous and locally bounded. The same statement holds for $\widehat{R}$ provided that an extra condition is satisfied. This extra condition corresponds to the assumption $\left(M 7_{X}\right)$ used below while replacing the set $X$ therein by $\mathbb{R}^{n}$. It was also shown that the map $R$ fails to be continuous with respect to time, as opposed to $\widehat{R}$ which enjoys this property provided that the latter extra condition is satisfied.

\section{PATHOLOGICAL CASES FOR LIPSCHITZNESS OF THE REACHABILITY MAPS}

When the system $\mathcal{H}_{f}$ in (11) with $C=\mathbb{R}^{n}$ and locally Lipschitz $F$ such that (A2) holds, using the well-known Filippov Theorem [5, Theorem 5.3.1], we are able to show that both maps $R^{b}\left(\equiv \widehat{R}^{b}\right)$ and $R(\equiv \widehat{R})$ are locally Lipschitz, see Lemma A.1. However, in the general case of hybrid systems satisfying the hybrid basic conditions (A1)(A4), even when the system is pre-complete with $F$ and $G$ single valued and smooth, the reachability maps $\left(R, R^{b}, \widehat{R}, \widehat{R}^{b}\right)$ may not be continuous at $\left(T_{o}, J, x_{o}\right) \in \mathbb{R}_{\geq 0} \times \mathbb{N} \times(C \cup D)$. Indeed, for the canonical bouncing-ball system, it is shown in [8, Example 4.4] that the maps $R$ and $R^{b}$ are continuous only at points $\left(T_{o}, J, x_{o}\right) \in$ $\mathbb{R}_{\geq 0} \times \mathbb{N} \times(C \cup D)$ such that the following condition holds:

$(\star \star)$ If $T_{o}>0$ then each solution $\phi$ starting from $x_{o}$ is such that, for each $j \in\{1,2, \ldots, J\} \cap \operatorname{dom} \phi$, its $j$-th jump does not occur at $\left(T_{o}, j-1\right)$.

In the sequel, we present different pathological scenarios of hybrid systems that, though satisfy (SA), their reachability maps $R$, $R^{b}, \widehat{R}$, and $\widehat{R}^{b}$ are not locally Lipschitz.

\subsection{When the Function $t_{\partial C}: C \rightarrow \mathbb{R}_{\geq 0}$ is not Locally Lipschitz on $S_{\partial C}$}

This scenario is illustrated in the following simple example.

EXAmple 3. Consider the constrained system $\mathcal{H}_{f}=(C, F)$ with

$$
F(x):=\left[\begin{array}{ll}
1 & 0
\end{array}\right]^{\top}, \quad C:=\left\{x \in \mathbb{R}^{2}: x_{1} \leq \sqrt{\left|x_{2}\right|}+2\right\} .
$$

Note that $F$ is single-valued and locally Lipschitz and that $\mathcal{H}_{f}$ is pre-complete. Moreover,

$$
t_{\partial C}(x)=t_{\partial C}^{\min }(x) \quad \forall x \in C .
$$

Furthermore, let $\left(x_{a o}, x_{b o}\right) \in C \times C$ with $x_{a o}:=\left[\begin{array}{ll}1 & 0\end{array}\right]^{\top}$ and $x_{b o}:=$ $\left[\begin{array}{ll}1 & \beta\end{array}\right]^{\top}$, for some $\beta \in[0,1]$. Let $\left(\phi_{a}, \phi_{b}\right)$ be the maximal solutions starting from $\left(x_{a o}, x_{b o}\right)$, respectively. After some computations, we obtain that $t_{\partial C}\left(x_{b o}\right)=t_{\partial C}^{\min }\left(x_{b o}\right)=1+\sqrt{\beta}, t_{\partial C}\left(x_{a o}\right)=t_{\partial C}^{\min }\left(x_{a o}\right)=$ $1, \phi_{b}\left(t_{\partial C}\left(x_{b o}\right), 0\right)=\left[\begin{array}{ll}2+\sqrt{\beta} & \beta\end{array}\right]^{\top}$, and $\phi_{a}\left(t_{\partial C}\left(x_{a o}\right), 0\right)=\left[\begin{array}{ll}2 & 0\end{array}\right]^{\top}$.

First, it is easy to see that the function $t_{\partial C}$ is not locally Lipschitz since $\left|t_{\partial C}\left(x_{b o}\right)-t_{\partial C}\left(x_{a o}\right)\right|=\sqrt{\beta}$ and $\left|x_{b o}-x_{a o}\right|=\beta$. Furthermore, for $T^{*}=2$, we obtain

$$
\tilde{R}^{b}\left(T^{*}, x_{b o}\right)=\phi_{2}\left(t_{\partial C}\left(x_{b o}\right), 0\right)
$$

and

$$
\tilde{R}^{b}\left(T^{*}, x_{a o}\right)=\phi_{1}\left(t_{\partial C}\left(x_{a o}\right), 0\right) .
$$

The latter implies that $x \mapsto R^{b}\left(T^{*}, x\right)$ is not locally Lipschitz on $C$ since

and

$$
\left|\tilde{R}^{b}\left(T^{*}, x_{b o}\right)-\tilde{R}^{b}\left(T^{*}, x_{a o}\right)\right|=\sqrt{\beta+\beta^{2}}
$$

$$
\left|x_{b o}-x_{a o}\right|=\beta
$$

\subsection{When Solutions are Nontrivial After Reaching $\partial C$}

Suppose the existence of a solution $\phi$ starting from $x_{o} \in \operatorname{int}(C)$ such that $0<t_{\partial C}\left(x_{o}\right)<\infty$ and $\operatorname{dom} \phi:=\left[0, T^{*}\right]$ with $T^{*}>t_{\partial C}$. In this case, it is possible to find an example where there exists a sequence of initial conditions $\left\{x_{o i}\right\}_{i=0}^{\infty} \subset \operatorname{int}(C)$ with $\lim _{i \rightarrow \infty} x_{o i}=$ $x_{o}$ such that each maximal solution $\phi_{i}$ staring from $x_{o i}$ satisfies $\operatorname{dom} \phi_{i}:=\left[0, t_{\partial C}\left(x_{o i}\right)\right]$ with $t_{\partial C}\left(x_{o i}\right) \leq t_{\partial C}\left(x_{o}\right)<T^{*}$. Hence, in such a scenario, the map $x \mapsto \tilde{R}^{b}\left(T^{*}, x\right)$ fails to be locally Lipschitz since, for each $i \in \mathbb{N}$,

$$
\left|\tilde{R}^{b}\left(T^{*}, x_{o}\right)-\tilde{R}^{b}\left(T^{*}, x_{o i}\right)\right|=\left|\phi\left(T^{*}, 0\right)-\phi_{i}\left(t_{\partial C}\left(x_{o i}\right), 0\right)\right| .
$$

In fact, the time mismatch in the right-hand side of the previous equality will not allow the map $\tilde{R}^{b}$ to be locally Lipschitz, see Example 4. 
EXAmPLe 4. Consider the constrained system $\mathcal{H}_{f}$ with

$$
F(x):=\left[\begin{array}{ll}
-1 & 0
\end{array}\right]^{\top}, C:=\mathbb{R}^{2} \backslash\left\{x \in \mathbb{R}^{2}: x_{1}<0,\left|x_{2}\right|<1\right\} .
$$

It is easy to see that (SA) is satisfied. Furthermore, let $\phi$ be the solution starting from $x_{o}:=\left[\begin{array}{ll}1 & 1\end{array}\right]^{\top}$, and let $\phi_{i}$ be the solution starting from $x_{o i}:=\left[\begin{array}{ll}1 & 1-(1 / i)\end{array}\right]^{\top}$. It is easy to see that $t_{\partial C}\left(x_{o i}\right)=$ $t_{\partial C}\left(x_{o}\right)=1,\left(t_{\partial C}\right.$ is locally Lipschitz $)$ and $\operatorname{dom} \phi_{i}=\left[0, t_{\partial C}\left(x_{o i}\right)\right]=$ $[0,1]$ for all $i \in \mathbb{N}$. However, dom $\phi=[0,+\infty)$; hence, when $T^{*}=2$ and for any $i \in \mathbb{N}$,

$$
\begin{aligned}
& \mid \tilde{R}^{b}\left(2, x_{o}\right)-\tilde{R}^{b}\left(2, x_{o i}\right)|=| \phi(2,0)-\phi_{i}(1,0) \mid \\
&=\left|\left[\begin{array}{ll}
-1 & 1
\end{array}\right]^{\top}-\left[\begin{array}{ll}
0 & 1-(1 / i)
\end{array}\right]^{\top}\right|>1,
\end{aligned}
$$

which shows that the map $x \mapsto \tilde{R}^{b}(2, x)$ is not locally Lipschitz.

\subsection{When Solutions Start From $\partial C$}

Suppose the existence of $x_{o} \in \partial C$ such that a nontrivial solution $\phi$ starting from $x_{o}$ exists; namely, $\operatorname{dom} \phi=\left[0, T^{*}\right]$, for some $T^{*}>0$. In this case, the following two situations prevent the maps $R$ and $R^{b}$ from being locally Lipschitz. The first situation is when there exists a sequence of initial conditions $\left\{x_{o i}\right\}_{i=0}^{\infty} \subset \partial C$ with $\lim _{i \rightarrow \infty} x_{o i}=x_{o}$ such that each maximal solution $\phi_{i}$ starting from $x_{o i}$ is trivial, i.e., $\operatorname{dom} \phi_{i}=\{0\}$. In such a scenario, the map $x \mapsto \tilde{R}^{b}\left(T^{*}, x\right)$ fails to be locally Lipschitz since

$$
\left|\tilde{R}^{b}\left(T^{*}, x_{o}\right)-\tilde{R}^{b}\left(T^{*}, x_{o i}\right)\right|=\left|\phi\left(T^{*}, 0\right)-\phi_{i}(0,0)\right|,
$$

and the time mismatch in the right-hand side of the previous equality will not allow the map to be locally Lipschitz. The following example illustrate this case.

ExAmple 5. Consider the constrained system $\mathcal{H}_{f}=(C, F)$ in Example 4 and let $x_{o}:=\left[\begin{array}{ll}0 & 1\end{array}\right]^{\top}$ and $x_{o i}:=\left[\begin{array}{ll}0 & 1-1 /(i+1)\end{array}\right]^{\top}$ for all $i \in \mathbb{N}$. It is easy to see in this case that (29) holds and reachability maps are not locally Lipschitz.

The second situation is when the solution $\phi$ starting from $x_{o} \in$ $\partial C$ remains in $\partial C$, its domain is unbounded, and there exists a sequence of initial conditions $\left\{x_{o i}\right\}_{i=0}^{\infty} \subset \partial C$ with $\lim _{i \rightarrow \infty} x_{o i}=x_{o}$ such that each maximal solution $\phi_{i}$ starting from $x_{o i}$ is nontrivial but its domain is bounded, i.e., $\operatorname{dom} \phi_{i}=\left[0, t_{\partial C}\left(x_{o i}\right)\right]$ and $\sup _{i \in \mathbb{N}}\left\{t_{\partial C}\left(x_{o i}\right)\right\}<\infty$. In such a scenario, the map $x \mapsto \tilde{R}^{b}\left(T^{*}, x\right)$ fails to be locally Lipschitz for sufficiently large $T^{*}>0$ since

$$
\left|\tilde{R}^{b}\left(T^{*}, x_{o}\right)-\tilde{R}^{b}\left(T^{*}, x_{o i}\right)\right|=\left|\phi\left(T^{*}, 0\right)-\phi_{i}\left(t_{\partial C}\left(x_{o i}\right), 0\right)\right| .
$$

This issue is illustrated in the following example.

EXAMPLE 6. Consider the constrained system $\mathcal{H}_{f}=(C, F)$ with

$$
\begin{gathered}
F(x):=\left[\begin{array}{ll}
1 & 0
\end{array}\right]^{\top} \quad \forall x \in C, \\
C:=\mathbb{R}^{2} \backslash\left\{x \in \mathbb{R}^{2}: x_{1} \in(1,2),\left|x_{2}\right|>0\right\} .
\end{gathered}
$$

Let $x_{o}:=\left[\begin{array}{ll}0 & 0\end{array}\right]^{\top}$ and let $x_{o i}:=\left[\begin{array}{ll}0 & 1 /(i+1)\end{array}\right]^{\top}$ for all $i \in \mathbb{N}$.

\subsection{When the Function $t_{D}: C \rightarrow \mathbb{R}_{\geq 0}$ is Not Locally Lipschitz on $S_{D}$}

Consider a compact set $K \subset \operatorname{int}(C)$ such that the following are satisfied:

(1) Each solution $\phi$ starting from $x_{o} \in K$ reaches the set $D$ after a time $t_{D}\left(x_{o}\right)>0$, where $t_{D}$ is as introduced in (14). Hence, $K \subset S_{D}$.
(2) There exists $T^{*} \in(0, \infty)$ such that

$$
T^{*}>\sup _{x_{o} \in K} t_{D}\left(x_{o}\right) \text {. }
$$

(3) After reaching the set $D$, each solution $\phi$ starting from $x_{o} \in K$ jumps back to the set $\operatorname{int}(C) \backslash D$.

(4) For every solution $\phi$ staring from $x_{o} \in K$,

$$
\left[t_{D}\left(x_{o}\right), T^{*}\right] \times\{1\} \subset \operatorname{dom} \phi .
$$

We start noticing that

$$
R^{b}\left(T^{*}, 1, x\right)=\widehat{R}^{b}\left(T^{*}, 1, x\right) \quad \forall x \in K
$$

and $\left(T^{*}, 1, x_{o}\right)$ satisfies $(\star \star)$ for all $x \in K$. Furthermore, if the map $x \mapsto R^{b}\left(T^{*}, 1, x\right)$ is locally Lipschitz on $K$, then there exists a constant $\lambda>0$ such that, for any two initial conditions $\left(x_{o 1}, x_{o 2}\right) \subset K \times K$ and two solutions $\left(\phi_{1}, \phi_{2}\right)$ starting from $\left(x_{o 1}, x_{o 2}\right)$, respectively,

$$
\left|R^{b}\left(T^{*}, 1, x_{o 1}\right)-R^{b}\left(T^{*}, 1, x_{o 2}\right)\right| \leq \lambda\left|x_{o 1}-x_{o 2}\right| .
$$

Now, using Lemma A.1 under (30), we conclude the existence of $\lambda_{1}>0$ such that

$$
\begin{gathered}
\left|R^{b}\left(T^{*}, 1, x_{o 1}\right)-R^{b}\left(T^{*}, 1, x_{o 2}\right)\right| \leq \lambda_{1}\left(\left|t_{D}\left(x_{o 1}\right)-t_{D}\left(x_{o 2}\right)\right|+\right. \\
\left.\left|\phi_{1}\left(t_{D}\left(x_{o 1}\right), 1\right)-\phi_{2}\left(t_{D}\left(x_{o 2}\right), 1\right)\right|\right) .
\end{gathered}
$$

Next, since the jump map is locally Lipschitz, we conclude the existence of $\lambda_{2}>0$ such that

$\left|\phi_{1}\left(t_{D}\left(x_{o 1}\right), 1\right)-\phi_{2}\left(t_{D}\left(x_{o 2}\right), 1\right)\right| \leq \lambda_{2}\left|\phi_{1}\left(t_{D}\left(x_{o 1}\right), 0\right)-\phi_{2}\left(t_{D}\left(x_{o 2}\right), 0\right)\right|$; hence,

$$
\begin{aligned}
\mid R^{b}\left(T^{*}, 1, x_{o 1}\right)- & R^{b}\left(T^{*}, 1, x_{o 2}\right)\left|\leq \lambda_{1}\right| t_{D}\left(x_{o 1}\right)-t_{D}\left(x_{o 2}\right) \mid+ \\
& \lambda_{1} \lambda_{2}\left|\phi_{1}\left(t_{D}\left(x_{o 1}\right), 0\right)-\phi_{2}\left(t_{D}\left(x_{o 2}\right), 0\right)\right| .
\end{aligned}
$$

Finally, using Lemma A.1, we conclude the existence of $\lambda_{3}$ such that

$$
\begin{aligned}
& \left|\phi_{2}\left(t_{D}\left(x_{o 2}\right), 0\right)-\phi_{1}\left(t_{D}\left(x_{o 1}\right), 0\right)\right| \leq \\
& \lambda_{3}\left(\left|t_{D}\left(x_{o 1}\right)-t_{D}\left(x_{o 2}\right)\right|+\left|x_{o 1}-x_{o 2}\right|\right) ;
\end{aligned}
$$

hence,

$$
\begin{aligned}
\mid R^{b}\left(T^{*}, 1, x_{o 1}\right)- & R^{b}\left(T^{*}, 1, x_{o 2}\right)\left|\leq \lambda_{1} \lambda_{2} \lambda_{3}\right| x_{o 1}-x_{o 2} \mid+ \\
& \left(\lambda_{1}+\lambda_{1} \lambda_{2} \lambda_{3}\right)\left|t_{D}\left(x_{o 1}\right)-t_{D}\left(x_{o 2}\right)\right| .
\end{aligned}
$$

In this particular scenario, when the minimal-time function $t_{D}$ is locally Lipschitz on $K$, we conclude that so is the map $x \mapsto$ $R^{b}\left(t^{*}, 1, x\right)$ on $K$. On the other hand, when the function $t_{D}$ is not locally Lipschitz on $K$, it is possible to construct a counterexample where $x \mapsto R^{b}\left(t^{*}, 1, x\right)$ is also not locally Lipschitz, as shown in the next example.

EXAMPLE 7. Consider the hybrid system with the following data:

$$
\begin{gathered}
F(x):=\left[\begin{array}{ll}
1 & 0
\end{array}\right]^{\top} \quad \forall x \in C:=\left\{x \in \mathbb{R}^{2}: x_{1} \geq \sqrt{\left|x_{2}\right|}+2\right\}, \\
G(x):=\left[\begin{array}{ll}
-x_{1} & x_{2}
\end{array}\right]^{\top} \quad \forall x \in D:=\mathbb{R}^{2} \backslash \operatorname{int}(C) .
\end{gathered}
$$

It easy to see that conditions (A1)-(A4) are satisfied, both $F$ and $G$ are locally Lipschitz, and that the maximal solutions to the systems are unique. Consider the compact set $K$ given by

$$
K:=\left\{x \in \mathbb{R}^{2}: x_{1}=1, x_{2} \in[0,1]\right\} .
$$

Let $\left(x_{o 1}, x_{o 2}\right) \in K \times K$ with $x_{o 1}:=\left[\begin{array}{ll}1 & 0\end{array}\right]^{\top}$ and $x_{o 2}:=\left[\begin{array}{ll}1 & \beta\end{array}\right]$, $\beta \in[0,1]$. After some computations, we obtain that

$$
t_{D}\left(x_{o 2}\right)=1+\sqrt{\beta}, \quad t_{D}\left(x_{o 1}\right)=1
$$


and

and

$$
\phi_{2}\left(t_{D}\left(x_{o 2}\right), 0\right)=\left[\begin{array}{ll}
2+\sqrt{\beta} & \beta
\end{array}\right]^{\top}
$$

$$
\phi_{1}\left(t_{D}\left(x_{o 1}\right), 0\right)=\left[\begin{array}{ll}
2 & 0
\end{array}\right]^{\top} .
$$

First, it is easy to see that the function $t_{D}$ is not locally Lipschitz since $\left|t_{D}\left(x_{o 2}\right)-t_{D}\left(x_{o 1}\right)\right|=\sqrt{\beta}$ and $\left|x_{o 2}-x_{o 1}\right|=\beta$. Furthermore, it is easy to see that $T^{*}=2$; hence,

$$
R^{b}\left(T^{*}, 1, x_{o 2}\right)=\left[\begin{array}{ll}
-1+2 \sqrt{\beta} & \beta
\end{array}\right]^{\top}
$$

and

$$
R^{b}\left(T^{*}, 1, x_{01}\right)=\left[\begin{array}{ll}
-1 & 0
\end{array}\right]^{\top} .
$$

The latter fact allows to conclude that the map $x \mapsto R^{b}\left(T^{*}, 1, x\right)$ is not locally Lipschitz since $\left|R^{b}\left(T^{*}, 1, x_{o 2}\right)-R^{b}\left(T^{*}, 1, x_{o 1}\right)\right|=\sqrt{4 \beta+\beta^{2}}$ and $\left|x_{02}-x_{1 o}\right|=\beta$.

\subsection{From Pure Discrete to Pure Continuous Behavior}

Suppose the existence of a solution $\phi$ starting from an initial condition $x_{o} \in C \cap D$ that jumps one time then flows. We assume further the existence of a sequence of initial conditions $\left\{x_{o i}\right\}_{i=0}^{\infty} \subset$ $D \backslash C$ with $\lim _{i \rightarrow \infty} x_{o i}=x_{o}$ such that each solution $\phi_{i}$ starting from $x_{o i}$ is a purely discrete solution that ends after only one jump. In this case, the possibility of flowing from $G\left(x_{o}\right)$ is an emergent behavior on the limit of the sequence $\left\{x_{o i}\right\}_{i=0}^{\infty}$, which may allow the solutions starting from $x_{o}$ to reach state values that are far enough from those reached from the $x_{o i}$ 's. As a consequence, the reachability maps in this case can fail to be locally Lipschitz around $x_{o}$, as shown in the next example.

EXAMPLE 8. Consider the hybrid system with the following data:

$$
\begin{array}{ll}
F(x):=\left[\begin{array}{ll}
-x_{2} & 1
\end{array}\right]^{\top} \quad \forall x \in C:=\left\{x \in \mathbb{R}^{2}: x_{1} \geq 0\right\}, \\
G(x):=\left[\begin{array}{ll}
x_{1} & -x_{2}
\end{array}\right]^{\top} \quad \forall x \in D:=\mathbb{R}_{\leq 0} \times \mathbb{R}_{\geq 0} .
\end{array}
$$

First, it easy to see that $R^{b}(1,1, x)=\widehat{R}^{b}(1,1, x)$ for all $x \in D$, and that $(1,1, x)$ satisfies $(\star \star)$ for all $x \in D$. Furthermore, we will show that the set-valued map $x \mapsto R^{b}(1,1, x)$ is not locally Lipschitz around the elements of the set $C \cap D:=\left\{x \in \mathbb{R}^{2}: x_{1}=0, x_{2} \geq 0\right\}$. Indeed, let $x_{o}:=\left[\begin{array}{ll}0 & 1\end{array}\right]^{\top}$ and let the sequence $\left\{x_{o i}\right\}_{i=0}^{\infty}$ given by $x_{o i}:=$ $\left[\begin{array}{ll}-1 / i & 1\end{array}\right]^{\top}$. It is easy to see that $R^{b}\left(1,1, x_{o i}\right)=\left\{\left[\begin{array}{ll}-1 / i & -1\end{array}\right]^{\top}\right\}$ for all $i \in \mathbb{N}$. Furthermore, the system admits a solution starting from $x_{o}=\left[\begin{array}{ll}0 & 1\end{array}\right]^{\top}$ given by $\phi(0,1)=\left[\begin{array}{ll}0 & -1\end{array}\right]^{\top}$ and $\phi(t, 1)=\left[(1 / 2) t^{2}-\right.$ $t \quad t-1]^{\top}$ for all $t \in[0,1]$. Hence, $R^{b}\left(1,1, x_{o}\right)=-\left[\begin{array}{ll}1 / 2 & 0\end{array}\right]^{\top}$. Hence, we conclude that $x \mapsto R^{b}(1,1, x)$ is not locally Lipschitz since $\left|R^{b}\left(1,1, x_{o i}\right)-R^{b}\left(1,1, x_{o}\right)\right|>1$ and $\left|x_{o i}-x_{o}\right|=1 / i$ for all $i \in \mathbb{N}$.

\subsection{From Pure Continuous to Pure Discrete Behavior}

Consider the solution $\phi$ starting from an initial condition $x_{o} \in$ $C \cap D$. Assume further the existence of a sequence of initial conditions $\left\{x_{o i}\right\}_{i=0}^{\infty} \subset C \backslash D$ with $\lim _{i \rightarrow \infty} x_{o i}=x_{o}$ such that each solution $\phi_{i}$ starting from $x_{o i}$ is either trivial or a pure continuous-time solution that never jump. In this case, the jump from $x_{o}$ is an emergent behavior on the limit of the sequence $\left\{x_{o i}\right\}_{i=0}^{\infty}$, which may allow the solutions starting from $x_{o}$ to reach new elements that are far enough from those reached from the $x_{o i}$ 's. As a consequence, the reachability maps, also in this case, can fail to be locally Lipschitz around $x_{o}$, as shown in the next example.
ExAmple 9. Consider the hybrid system with the following data:

$$
\begin{gathered}
F(x):=\left[\begin{array}{ll}
-1 & \left|x_{1}\right|
\end{array}\right]^{\top} \quad \forall x \in C:=\mathbb{R}^{2} \backslash\left\{x \in \mathbb{R}^{2}: x_{1}<0, x_{2}>0\right\}, \\
G(x):=\left[\begin{array}{ll}
0 & x_{2}-1
\end{array}\right]^{\top} \quad \forall x \in D:=\left\{x \in \mathbb{R}^{2}: x_{2}=0, x_{1} \leq 0\right\} .
\end{gathered}
$$

First, it easy to see that $R^{b}(1,1, x)=\widehat{R}^{b}(1,1, x)$ for all $x \in D$ and that $(1,1, x)$ satisfies $(\star \star)$ for all $x \in D$. Furthermore, we will show that the set-valued map $x \mapsto R^{b}(1,1, x)$ is not locally Lipschitz around the element $x_{o}=\left[\begin{array}{ll}0 & 0\end{array}\right]^{\top} \in C \cap D=\left\{x \in \mathbb{R}^{2}: x_{1}=0, x_{2} \geq 0\right\}$. Indeed, let the sequence $\left\{x_{o i}\right\}_{i=0}^{\infty}$ given by $x_{o i}:=\left[\begin{array}{ll}x_{o i 1} & x_{o i 2}\end{array}\right]^{\top}:=$ $\left[\begin{array}{ll}1 /(i+1) & 0\end{array}\right]^{\top}$. It is easy to see that $R^{b}\left(1,1, x_{o i}\right)=\phi_{i}\left(t=x_{o i 1}, 0\right)=$ $\left[\begin{array}{ll}0 & x_{\text {oi } 1}^{2} / 2\end{array}\right]^{\top}$ for all $i \in \mathbb{N}$. Furthermore, the system admits a solution starting from $x_{o}$ given by $\phi(0,1)=\left[\begin{array}{ll}0 & -1\end{array}\right]^{\top}$ and $\phi(t, 1)=$ $\left[\begin{array}{ll}-t & -t^{2} / 2-1\end{array}\right]^{\top}$ for all $t \in[0,1]$. Hence, $R^{b}\left(1,1, x_{o}\right)=[-1 \quad-$ $3 / 2]^{\top}$, which implies that $x \mapsto R^{b}(1,1, x)$ is not locally Lipschitz around $\left(1,1, x_{o}\right)$ since $\left|R^{b}\left(1,1, x_{o i}\right)-R^{b}\left(1,1, x_{o}\right)\right|>1$ and $\left|x_{o i}-x_{o}\right|=$ $1 /(i+1)$ for all $i \in \mathbb{N}$.

\section{SUFFICIENT CONDITIONS FOR LIPSCHITZNESS OF REACHABILITY MAPS}

In this section, we investigate sufficient conditions on $\mathcal{H}$ guaranteeing that the reachability maps $\left(R, R^{b}, \widehat{R}, \widehat{R}^{b}\right)$ are locally Lipschitz (in the sense of Definition 3 ) in some regions within their domain. For starters, we assume

(SA) The system $\mathcal{H}=(C, F, D, G)$ is such that (A1) holds, $F$ is single valued and locally Lipschitz on $C$, and $G$ is single valued and locally Lipschitz on $D$. Furthermore, $\mathcal{H}$ is pre-complete and has unique solutions.

These properties are easy to check. In particular, completeness and uniqueness are satisfied when the flows of $\mathcal{H}$ do not escape in finite time and the conditions in [12, Proposition 2.11] hold. It is important to notice that the hybrid systems in the examples presented in Section 4 do satisfy (SA). In addition to (SA), and to avoid the scenarios in Sections 4.1-4.6, we consider the following additional assumptions with respect to a given set $X \subset \mathbb{R}^{n}$ :

$\left(\mathrm{M} 1_{X}\right)$ The set $X$ is forward pre-invariant for $\mathcal{H}$; namely, $\phi(\operatorname{dom} \phi) \subset$ $X$ for all $\phi \in \hat{\mathcal{S}}_{\mathcal{H}}(X)$.

$\left(\mathrm{M} 2_{X}\right)$ The function $t_{\partial C}$ defined in (14) is locally Lipschitz on $S_{\partial C} \cap$ $X$, where $S_{\partial C}$ is given in (16).

$\left(\mathrm{M} 3_{X}\right)$ The flows of $\mathcal{H}$ starting from $x_{o} \in X \cap \partial C$, with $x_{o}$ reachable by a flow starting from some point $y_{o} \in X \backslash\left\{x_{o}\right\}$, are trivial.

$\left(\mathrm{M}_{X}\right)$ For any $x_{o} \in \partial C \cap X$ from which a nontrivial flow of $\mathcal{H}$ exists, there exists $U\left(x_{o}\right) \subset \mathbb{R}^{n}$ such that the following holds:

$\forall \psi \in \hat{\mathcal{S}}_{\mathcal{H}}\left(U\left(x_{o}\right) \cap X \cap \partial C\right), \exists t_{\psi}>0: \psi\left(\left(0, t_{\psi}\right], 0\right) \subset \operatorname{int}(C)$.

$\left(\mathrm{M}_{X}\right)$ The function $t_{D}$ is locally Lipschitz on $S_{D} \cap X$.

$\left(\mathrm{M}_{X}\right)$ For any $x_{o} \in C \cap D \cap X$, the following hold:

(a) If $G\left(x_{o}\right) \in C \cup D$, then there exists $U\left(x_{o}\right)$ such that

$$
G(x) \in C \cup D \quad \forall x \in U\left(x_{o}\right) \cap D \cap X .
$$

(b) There exists $U\left(x_{o}\right)$ such that

$$
t_{D}(x)<\infty \quad \forall x \in U\left(x_{o}\right) \cap(C \backslash D) \cap X .
$$

Remark 5. Assumptions $\left(M 1_{X}\right)-\left(M 6_{X}\right)$ are imposed in the results in this section to handle the pathological scenarios in Sections 4.1-4.6. In particular, when $\left(\mathrm{M} 2_{X}\right)$ and $\left(\mathrm{M} 5_{X}\right)$ hold, the scenarios in Sections 4.1 and 4.4 cannot happen, respectively. Furthermore, 
when $\left(\mathrm{M} 3_{X}\right)$ holds, the pathological behavior in Section 4.2 cannot occur either. Also, when $\left(M 4_{X}\right)$ holds, the scenario in Section 4.3 cannot happen. Finally, when $\left(\mathrm{M}_{X}\right)(\mathrm{a})$ and $\left(\mathrm{M}_{X}\right)(\mathrm{b})$ hold, the scenarios in Sections 4.5 and 4.6 cannot occur either, respectively. $\bullet$

REMARK 6. To verify $\left(\mathrm{M}_{X}\right)$ and $\left(\mathrm{M}_{X}\right)$, necessary and sufficient infinitesimal conditions can be found in [18, Theorem 1]. Furthermore, Lemmas A.2 and A.3 provide sufficient infinitesimal conditions to verify $\left(\mathrm{M}_{X}\right)$ and $\left(\mathrm{M}_{X}\right)$, respectively.

\subsection{Local Lipschitzness of $R$ and $R^{b}$}

In the following result, we show that for the class of hybrid systems satisfying (SA) and $\left(\mathrm{M}_{X}\right)-\left(\mathrm{M} 6_{X}\right)$ with respect to $X \subset \mathbb{R}^{n}$, the maps $(T, x) \mapsto R^{b}(T, J, x)$ and $(T, x) \mapsto R(T, J, x)$ are locally Lipschitz around each $\left(T_{o}, J, x_{o}\right) \in \mathbb{R}_{\geq 0} \times \mathbb{N} \times(X \cap(C \cup D))$ provided that condition $(\star \star)$ given at the beginning of Section 4 holds. Condition $(\star \star)$ was used in [8] to show continuity of $(T, x) \mapsto R^{b}(T, J, x)$ and $(T, x) \mapsto R(T, J, x)$ with respect to $T$ around $\left(T_{o}, J, x_{o}\right)$.

Theorem 5.1. Consider a hybrid system $\mathcal{H}=(C, F, D, G)$ such that (SA) holds and a set $X \subset \mathbb{R}^{n}$ such that $\left(M 1_{X}\right)-\left(M 6_{X}\right)$ hold. Then, the maps $R^{b}$ and $R$ in (19) and (20), respectively, are locally Lipschitz around each $\left(T_{\boldsymbol{o}}, J, x_{\boldsymbol{o}}\right) \in \mathbb{R}_{\geq 0} \times \mathbb{N} \times(X \cap(C \cup D))$ satisfying ( $\left.\star \star\right)$.

\subsection{Local Lipschitzness of $\widehat{R}$ and $\widehat{R}^{b}$}

When the following extra condition holds, we show that the maps $\widehat{R}^{b}$ and $\widehat{R}$ are locally Lipschitz on $\mathbb{R}_{\geq 0} \times \mathbb{N} \times(X \cap(C \cup D))$ :

$\left(M 7_{X}\right)$ If the (unique) maximal solution $\phi$ starting from $x_{o} \in C \cap X$ never jumps, then, there exists $U\left(x_{o}\right)$ such that, for every $y_{o} \in U\left(x_{o}\right) \cap X \cap C$, the maximal solution $\psi$ starting from $y_{o}$ never jumps.

In the following example, to highlight the need of condition $\left(M 7_{X}\right)$, we construct a hybrid system where $(\mathrm{SA})$ and $\left(\mathrm{M} 1_{X}\right)-\left(\mathrm{M} 6_{X}\right)$ are satisfied with $X=\mathbb{R}^{n}$ but $\left(M 7_{X}\right)$ does not hold. Since the conditions in Theorem 5.2 do not hold, the map $x \mapsto \widehat{R}^{b}\left(T_{o}, 1, x\right)$ is not locally Lipschitz for some $T_{o}>0$, as we show in the example.

EXAMPLE 10. Consider the hybrid system $\mathcal{H}$ with the following data:

$$
\begin{array}{cc}
F(x):=\left[\begin{array}{ll}
x_{1}^{2} & 1
\end{array}\right]^{\top} \quad \forall x \in C:=\left\{x \in \mathbb{R}^{2}:\left|x_{1}\right| \leq 1, x_{2} \geq 0\right\}, \\
G(x):=\left[\begin{array}{ll}
-x_{1} & x_{2}
\end{array}\right]^{\top} \quad \forall x \in D:=\left\{x \in \mathbb{R}^{2}: x_{1}=1, x_{2} \geq 0\right\} .
\end{array}
$$

The flows starting from initial conditions $x_{o} \in C \backslash D$ are given by

$$
\phi_{2}(t, 0)=x_{o 2}+t, \quad \phi_{1}(t, 0)=\frac{x_{o 1}}{1-x_{o 1} t} .
$$

We notice that the solutions to $\mathcal{H}$ starting from

$$
C_{1}:=\left\{x \in \mathbb{R}^{2}: x_{1} \in[-1,0], x_{2} \geq 0\right\}
$$

never jump and the maximal solutions are complete. Furthermore, the maximal solutions starting from

$$
C_{2}:=\left\{x \in \mathbb{R}^{2}: x_{1} \in(0,1], x_{2} \geq 0\right\}
$$

flow until reaching the set $D$, from which all the jumps take the solutions instantaneously to the set $C_{1}$. The latter fact implies that the maximal solutions to $\mathcal{H}$ are complete and unique; hence, (SA) is satisfied. In order to show that $\left(\mathrm{M} 2_{X}\right)-\left(\mathrm{M} 6_{X}\right)$ are satisfied, we notice that $S_{D}=C_{2}$ and that $t_{D}\left(x_{o}\right)=\left(1 / x_{o 1}\right)-1$ for all $x_{o} \in S_{D}$, which is a $C^{1}$ function on $S_{D}$; thus, locally Lipschitz. Hence, $\left(\mathrm{M} 5_{X}\right)$ is satisfied. Next, we notice that $S_{\partial C}=C_{2}=S_{D}$ and, for all $x_{o} \in S_{\partial C}, t_{\partial C}\left(x_{o}\right)=t_{D}\left(x_{o}\right)$ which is locally Lipschitz. Hence,
$\left(\mathrm{M} 2_{X}\right)$ is satisfied. Furthermore, we notice that the solutions flowing from $\operatorname{int}(C)$ and reaching $\partial C$, they also reach the set $D$ at the same time and can only jump from $D$. Hence, $\left(\mathrm{M}_{3_{X}}\right)$ is satisfied. Moreover, all the nontrivial flows starting from $\partial C$ flow instantaneously to the interior of $C$ according to (34). Hence, $\left(M 4_{X}\right)$ is satisfied. Finally, we notice that $C \cap D=\left\{x \in \mathbb{R}^{2}: x_{1}=1, x_{2} \geq 0\right\}$ and that $U(C \cap D) \cap(C \backslash D) \subset C_{2}=S_{D}$; hence, $\left(\mathrm{M}_{X}\right)(\mathrm{b})$ is satisfied. Also, $G\left(x_{o}\right) \in C \backslash D$ for all $x_{o} \in D$. Hence, $\left(M 6_{X}\right)((\mathrm{a}))$ is satisfied. On the other hand, we will show that $\left(M 7_{X}\right)$ is not satisfied. Indeed, the maximal solution starting from $x_{o}=\left[\begin{array}{ll}0 & 0\end{array}\right]^{\top}$ never jumps and is complete. However, each solution in the sequence of solutions $\left\{\phi_{i}\right\}_{i=1}^{\infty}$, starting from $\left\{x_{o i}\right\}_{i=1}^{\infty}$, respectively, with $x_{o i}:=$ $[1 /(i+2) \quad 0]^{\top}$ for all $i \in\{1, \ldots, \infty\}$ and $\lim _{i \rightarrow \infty} x_{o i}=x_{o}$, jumps at $t_{D}\left(x_{o i}\right)=(i+1,0)$. Furthermore, according to (26), we conclude that $\widehat{R}^{b}\left(1,1, x_{o}\right)=\left[\begin{array}{ll}0 & 1\end{array}\right]^{\top}$ and that $\widehat{R}^{b}\left(1,1, x_{o i}\right)=\left[\begin{array}{ll}1 & 1+i\end{array}\right]^{\top}$. Hence, $\left|\widehat{R}^{b}\left(1,1, x_{o}\right)-\widehat{R}^{b}\left(1,1, x_{o i}\right)\right|=|[1 \quad i]| \geq 1$, which shows that $x \mapsto \widehat{R}^{b}(1,1, x)$ is not locally Lipschitz around the origin.

Theorem 5.2. Consider a hybrid system $\mathcal{H}=(C, F, D, G)$ such that (SA) holds and a set $X \subset \mathbb{R}^{n}$ such that $\left(M 1_{X}\right)-\left(M 7_{X}\right)$ hold. Then, the maps $\widehat{R}^{b}$ and $\widehat{R}$ in (21) and (26), respectively, are locally Lipschitz on $\mathbb{R}_{\geq 0} \times \mathbb{N} \times(X \cap(C \cup D))$.

\subsection{Examples}

In the following example, we illustrate Theorems 5.1 and 5.2 on the bouncing ball hybrid model.

EXAMPLE 11. [Bouncing ball] Let us reconsider the dynamical hybrid model of the bouncing ball system in Example 2. It is easy to see that the system's solutions are unique, the flow and the jump maps $F$ and $G$ are both single valued and locally Lipschitz, and the system's solutions are complete; hence, (SA) is satisfied. Furthermore, we will show that the additional conditions $(\mathrm{M} 3 X)$, $\left(\mathrm{M}_{X}\right)$, and $\left(\mathrm{Mb}_{X}\right)$ are also satisfied for all $X \subset \mathbb{R}^{n}$. However, $\left(\mathrm{M} 2_{X}\right)$ and $\left(\mathrm{M}_{X}\right)$ are satisfied for all $X \subset \mathbb{R}^{n} \backslash\{0\}$. Indeed, we start noticing that the nontrivial solutions flowing from $\partial C$ are only those starting from the set $\partial C \backslash D=\left\{x \in \mathbb{R}^{2}: x_{1}=0, x_{2}>0\right\}$. Furthermore, since the set $C$ is convex thus regular, using the second statement in Lemma A.2, we conclude that the elements of the set $\partial C \backslash D$ from which a nontrivial flow exists cannot be reached by the system's flows that start from $\operatorname{int}(C)$; hence, $\left(\mathrm{M}{ }_{X}\right)$ is satisfied. Next, the nontrivial solution $\phi$ starting from $x_{o} \in \partial C \backslash D$ satisfies $\phi(t)=\left[\begin{array}{ll}-\frac{1}{2} \gamma t^{2}+x_{o 2} t & -\gamma t+x_{o 2}\end{array}\right]^{\top}$ for all $t \geq 0$, which implies that $\phi_{1}(t)>0$ for all $t \in\left(0,2 x_{o 2} / \gamma\right)$; thus, $\phi(t) \in \operatorname{int}(C)$ for all $t \in\left(0,2 x_{o 2} / \gamma\right)$, which satisfies $\left(\mathrm{M}_{X}\right)$. Another way to verify $\left(\mathrm{M}_{X}\right)$, consists in using Lemma A.3. Indeed, the set $C$ is regular and for all $x_{o} \in \partial C \backslash D, F\left(x_{o}\right) \in D_{C}\left(x_{o}\right)$. Also, we notice that, for all $x_{o} \in D$, $G\left(x_{o}\right) \in C \cup D$, which implies that $\left(\mathrm{M}_{X}\right)(\mathrm{a})$ is satisfied. Finally, since for any initial condition $x_{o} \in C, t_{D}\left(x_{o}\right)<\infty,\left(\mathrm{M}_{X}\right)(\mathrm{b})$ is also satisfied. Furthermore, note that $\partial C=\left\{x \in \mathbb{R}^{2}: x_{1}=0\right\}$ and $D=$ $\left\{x \in \mathbb{R}^{2}: x_{1}=0, x_{2} \leq 0\right\}$. Hence, using Definition 6, we conclude that $S_{\partial C}=S_{D}=C$. Indeed, from any element $x_{o} \in C$, either there exists a nontrivial solution to (11) that reaches $\partial C$ and $D$ at the same time, otherwise, the solution starts from $\partial C \cap D$ is trivial. Also, according to Definition 6 and after some easy computations, we conclude that $t_{\partial C}\left(x_{o}\right)=t_{D}\left(x_{o}\right)=\left(x_{o 2}+\sqrt{x_{o 2}^{2}+2 \gamma x_{o 1}}\right) / \gamma$ for all $x \in C$. Hence, $t_{\partial C}$ and $t_{D}$ are $C^{1}$ everywhere (thus, locally Lipschitz) except at the origin, which implies that $\left(\mathrm{M} 2_{X}\right)$ and $\left(\mathrm{M} 5_{X}\right)$ hold for each $X \subset \mathbb{R}^{n} \backslash\{0\}$. The latter is confirmed in [18, Example 
4] using the infinitesimal conditions proposed in the latter reference. Moreover, since the system's maximal solutions cannot only flow, then $\left(M 7_{X}\right)$ is trivially satisfied with respect to any $X \subset \mathbb{R}^{n}$. Finally, since the origin cannot be reached by the maximal solutions starting from an initial point different than the origin, we conclude that the candidate set $X:=C \backslash\{0\}$ is forward pre-invariant, i.e., it satisfies $\left(\mathrm{M} 1_{X}\right)$. As a result, Theorem 5.1 can be used to conclude that the maps $R^{b}$ and $R$ are locally Lipschitz around each $\left(T_{o}, J, x_{o}\right) \in \mathbb{R}_{\geq 0} \times$ $\mathbb{N} \times(X \cap(C \cup D))$ satisfying $(\star \star)$. Moreover, using Theorem 5.2, we can also conclude that the maps $\widehat{R}^{b}$ and $\widehat{R}$ are locally Lipschitz on $\mathbb{R}_{\geq 0} \times \mathbb{N} \times(X \cap(C \cup D))$.

In many examples of hybrid systems encountered in applications, the interior of the set $C$ is empty. This is the case, for example, when the state $x$ contains discrete variables. For such a class of hybrid systems, we notice that the statements in Theorems 5.1 and 5.2 are not directly applicable as $\left(\mathrm{M} 4_{X}\right)$ cannot be verified when $\operatorname{int}(C)=\emptyset$. However, it is possible to handle this situation by introducing an extended hybrid system $\mathcal{H}_{e}=\left(C_{e}, F, D_{e}, G\right)$ where the discrete state variables are allowed to have a continuous evolution. Furthermore, we choose the set $X$ such that the solutions to $\mathcal{H}_{e}$ restricted to $X$ are the solutions to the original system $\mathcal{H}$. By doing so, the set $C_{e}$ will have a nonempty interior and $\left(\mathrm{M}_{X}\right)$ can be verified for $\mathcal{H}_{e}$ with respect $X$ and Theorems 5.1 and 5.2 become applicable. This approach is illustrate in Example 12.

EXAMPLE 12. [Thermostat] Consider the hybrid model of the thermostat system proposed in [12, Example 1.9] and given by $\mathcal{H}=(C, F, D, G)$ with $x:=\left[\begin{array}{ll}q & z\end{array}\right]^{\top} \in \mathbb{R}^{2}$,

$$
\begin{aligned}
F(x) & :=\left[\begin{array}{ll}
0 & -z+z_{0}+z_{\Delta} q
\end{array}\right]^{\top}, C:=\left(\{0\} \times C_{0}\right) \cup\left(\{1\} \times C_{1}\right), \\
C_{0} & :=\left\{z: z \geq z_{\min }\right\}, C_{1}:=\left\{z: z \leq z_{\max }\right\}, \\
G(x) & :=\left[\begin{array}{ll}
1-q & z
\end{array}\right]^{\top}, D:=\left(\{0\} \times D_{0}\right) \cup\left(\{1\} \times D_{1}\right), \\
D_{0} & :=\left\{z: z \leq z_{\min }\right\}, D_{1}:=\left\{z: z \geq z_{\max }\right\} .
\end{aligned}
$$

where $z$ is the temperature of the room, $z_{o}$ represents the natural temperature of the room when the heater is not used, $z_{\Delta}$ the capacity of the heater to raise the temperature in the room by always being on, and $q$ the state of the heater, which is 1 (on) or 0 (off). We want to keep the temperature between $z_{\min }$ and $z_{\max }$ satisfying $z_{o}<z_{\min }<z_{\max }<z_{o}+z_{\Delta}$. It is easy to see that the system's solutions flow only on the boundary of the set $C$; hence, $\left(\mathrm{M}_{X}\right)$ cannot be verified. In order to handle this situation, we propose to add more solutions to the hybrid model $\mathcal{H}$ by modifying the sets $C$ and $D$ and allowing the discrete variable $q$ to have not only the discrete values $\{0,1\}$. That is, we introduce the extended hybrid system $\mathcal{H}_{e}=\left(C_{e}, F, D_{e}, G\right)$ with the data

$$
\begin{aligned}
x & :=\left[\begin{array}{ll}
q & z
\end{array}\right]^{\top} \in \mathbb{R}^{2}, F(x):=\left[\begin{array}{ll}
0 & -z+z_{o}+z_{\Delta} q
\end{array}\right]^{\top}, \\
C_{e} & :=\left([-\epsilon, \epsilon] \times C_{0}\right) \cup\left([1-\epsilon, 1+\epsilon] \times C_{1}\right), \\
G(x) & :=\left[\begin{array}{ll}
1-q & z
\end{array}\right]^{\top}, D_{e}:=\left([-\epsilon, \epsilon] \times D_{0}\right) \cup\left([1-\epsilon, 1+\epsilon] \times D_{1}\right),
\end{aligned}
$$

for $\epsilon>0$ sufficiently small. Furthermore, we consider the set $X:=$ $\{0,1\} \times \mathbb{R}$. Note that the $X$ is forward pre-invariant for $\mathcal{H}_{e}$ and the solutions to $\mathcal{H}$ starting from $X$ are solutions to $\mathcal{H}_{e}$. Hence, showing that the proposed reachability maps for $\mathcal{H}_{e}$ are locally Lipschitz on $\mathbb{R}_{\geq 0} \times \mathbb{N} \times\left(X \cap\left(C_{e} \cup D_{e}\right)\right)$ is enough to conclude that the reachbility maps for $\mathcal{H}$ are locally Lipschitz on $\mathbb{R}_{\geq 0} \times \mathbb{N} \times(C \cup D)$. Hence, it remains to show that $(\mathrm{SA})$ and $\left(\mathrm{M} 2_{X}\right)-\left(\mathrm{M} 7_{X}\right)$ hold with respect to $X$ for the extended hybrid system $\mathcal{H}_{e}$. Indeed, the solutions of $\mathcal{H}_{e}$ starting from the set $X$ are the solutions to $\mathcal{H}$; hence, they are unique and complete. Moreover, it is clear that the system
$\mathcal{H}_{e}$ has unique solutions and is well posed, and both $F$ and $G$ are locally Lipschitz; thus, (SA) is satisfied. Furthermore, we notice $S_{\partial C_{e}} \cap K=S_{D_{e}} \cap K=C$, and

$$
t_{\partial C_{e}}(x)=t_{D_{e}}(x)=\left\{\begin{array}{cl}
-\log \frac{z_{\min }-z_{o}}{z-z_{o}} & \text { if } x \in\{0\} \times C_{o} \\
-\log \frac{z_{\max }-z_{o}-z_{\Delta}}{z-z_{o}-z_{\Delta}} & \text { if } x \in\{1\} \times C_{1},
\end{array}\right.
$$

which is locally Lipschitz; hence, $\left(\mathrm{M} 2_{X}\right)$ and $\left(\mathrm{M} 5_{X}\right)$ hold with respect to $X$. Next, since each solution starting from the set $X$ jumps once reaching $\partial C_{e}$ and since the solutions are unique; hence, the flow is not possible after reaching $\partial C_{e}$, we conclude that $\left(\mathrm{M}{ }_{X}\right)$ holds with respect to $X$. Moreover, $\left(M 4_{X}\right)$ holds trivially with respect to $X$ since the flows starting from $\partial C_{e} \cap X=\emptyset$ are trivial. Furthermore, $C_{e} \cap D_{e} \cap X=\left\{\left(1, z_{\text {max }}\right)\right\} \cup\left\{\left(0, z_{\text {min }}\right)\right\}$ and, for all $x_{o} \in C_{e} \cap D_{e} \cap X$, we can find $U\left(x_{o}\right)$ nonempty such that $U\left(x_{o}\right) \cap D \cap X=\emptyset$; hence, $\left(\mathrm{M}_{X}\right)(\mathrm{a})$ is satisfied trivially with respect to $X$. Moreover, since we have already shown that $C_{e} \cap X=C \subset S_{D_{e}}$, it follows that $\left(M 6_{X}\right)(\mathrm{b})$ holds with respect to $X$. Finally, using the fact that $C_{e} \cap X=C \subset S_{D_{e}}$, we conclude that all the maximal solutions starting from $X$ are not pure flows; hence, $\left(\mathrm{M} 7_{X}\right)$ holds trivially with respect to $X$.

Hence, Theorem 5.1 can be used to conclude that the maps $R^{b}$ and $R$ are locally Lipschitz around each $\left(T_{o}, J, x_{o}\right) \in \mathbb{R}_{\geq 0} \times \mathbb{N} \times(C \cup D)$ satisfying $(\star \star)$. Moreover, using Theorem 5.2 , we can also conclude that the maps $\widehat{R}^{b}$ and $\widehat{R}$ are locally Lipschitz on $\mathbb{R}_{\geq 0} \times \mathbb{N} \times(C \cup D)$.

\section{APPLICATION TO SAFETY}

\subsection{Safety Analysis Using Barrier Functions}

Given a hybrid system $\mathcal{H}=(C, F, G, D)$ and two sets $X_{o} \subset$ $\operatorname{cl}(C) \cup D$ and $X_{u} \subset \mathbb{R}^{n}$, the hybrid system $\mathcal{H}$ is safe with respect to $\left(X_{o}, X_{u}\right)$, with $X_{o} \cap X_{u}=\emptyset$, if the solutions starting from $X_{o}$ never reach the set $X_{u}$; namely, each maximal solution $\phi$ starting from $x_{o} \in X_{o}$ satisfies $\phi(t, j) \in \mathbb{R}^{n} \backslash X_{u}$ for all $(t, j) \in \operatorname{dom} \phi$. The points not in $\operatorname{cl}(C) \cup D$ are considered unsafe, which implies that $\mathbb{R}^{n} \backslash(\operatorname{cl}(C) \cup D) \subset X_{u}$. Furthermore, a barrier function candidate with respect to the sets $\left(X_{o}, X_{u}\right)$ is defined as a scalar function $B: \operatorname{cl}(C) \cup D \rightarrow \mathbb{R}$ such that

$$
\begin{array}{cc}
B(x)>0 & \forall x \in X_{u} \cap(\operatorname{cl}(C) \cup D) \\
B(x) \leq 0 & \forall x \in X_{o} .
\end{array}
$$

A barrier function candidate $B$ allows to conclude safety if it allows to conclude that the set $K:=\{x \in \operatorname{cl}(C) \cup D: B(x) \leq 0\}$ is closed and forward pre-invariant; namely, the maximal solutions to $\mathcal{H}$ starting from $K$ stay in $K$, see [20, Theorem 3.2]. In turn, the set $K$ is forward pre-invariant if the solutions starting from $K$ do not jump outside $K$; namely, for all $x \in K \cap D$,

$$
G(x) \subset \operatorname{cl}(C) \cup D \quad \text { and } \quad B(\eta) \leq 0 \quad \forall \eta \in G(x)
$$

and the solutions flowing from the set $K$ never leave $K$. To conclude the latter property, it is enough to show that condition $(\star)$ given in Section 2.4 holds with the set $C$ therein replaced by $C \backslash \operatorname{int}(K)$.

The converse safety problem consists in showing the existence of a barrier function $B$ such that the properties (35), (36), and ( $\star$ ) are satisfied provided that the system $\mathcal{H}$ is safe with respect to $\left(X_{o}, X_{u}\right)$. One of the challenges when studying the converse safety problem is to show the existence of a barrier function with the best possible smoothness property. Indeed, the availability of a smooth barrier function allows to characterize $(\star)$ using infinitesimal conditions involving only the set $C$ and the map $F$, as in Lemma 2.1, instead of any knowledge about the solutions. It is shown in [9] that a 
safe differential equation with smooth right-hand side does not guarantee the existence of a smooth autonomous barrier function satisfying (35), (36), and ( $\star$ ) that is also continuous. As a consequence, in [20] and [8], non-autonomous barrier-like functions are introduced to assure that a hybrid system $\mathcal{H}$ that is safe with respect to $\left(X_{o}, X_{u}\right)$ with $X_{o}$ closed is equivalent to the existence of $B: \mathbb{R}_{\geq 0} \times \mathbb{N} \times(C \cup D) \rightarrow \mathbb{R}$ satisfying properties, similar to (35), $(36)$, and $(\star)$, that are sufficient for safety.

\subsection{Locally Lipschitz Barrier Functions for a Class of Safe Hybrid Systems}

The barrier function constructed in [8] for a safe, with respect to $\left(X_{o}, X_{u}\right)$, hybrid system $\mathcal{H}=(C, F, G, D)$ depends on the reachability map $\widehat{R}$ for the backward in time hybrid system $\mathcal{H}^{-}=\left(C,-F, G(D), G_{D}^{-}\right)$and is precisely given by

$$
B(T, J, x):=\inf _{y \in \widehat{R}_{b}(T, J, x)}|y|_{X_{o}}
$$

for all $(T, J, x) \in \mathbb{R}_{\geq 0} \times \mathbb{N} \times(\operatorname{cl}(C) \cup D)$, where

$$
\begin{aligned}
\widehat{R}_{b}(T, J, x): & \widehat{R}(-T,-J, x)= \\
& \left\{\phi(t, j): \phi \in \hat{\mathcal{S}}_{\mathcal{H}}^{-}\left(x_{o}\right),(t, j) \in \operatorname{dom} \phi \cap \mathcal{T}_{\phi}(T, J)\right\},
\end{aligned}
$$

with $\mathcal{T}_{\phi}(T, J)$ as introduced in $(22), \hat{\mathcal{S}}_{\mathcal{H}}^{-}$as in Section 2.2, and $\widehat{R}$ as in (21).

It is shown in [8, Theorem 5.4] that the barrier function $B$ in (37) is lower semicontinuous with respect to $x$ and continuous with respect to $T$ (the ordinary (flow) time) provided that (A1)-(A2) hold, $\mathcal{H}^{-}$is pre-complete, the solutions to $\mathcal{H}^{-}$satisfy $\left(\mathrm{M} 7_{X}\right)$ (with $X=C \cup G(D)$ ), and the reciprocal jump map $G_{D}^{-1}: G(D) \rightrightarrows \mathbb{R}^{n}$ in (10) is outer semicontinuous and locally bounded. In the following result and for hybrid systems $\mathcal{H}$ such that $\mathcal{H}^{-}$satisfies (SA) and $\left(\mathrm{M} 1_{X}\right)-\left(\mathrm{M} 7_{X}\right)$ with respect to $X \subset \mathbb{R}^{n}$, using Theorem 5.2 , we conclude that the barrier candidate $B$ in (37) is locally Lipschitz on $\mathbb{R}_{\geq 0} \times \mathbb{N} \times(X \cap(C \cup G(D)))$. Furthermore, the same holds on $\mathbb{R}_{\geq 0} \times \mathbb{N} \times(X \cap(C \cup D))$ when, additionally,

$$
\operatorname{cl}(D \backslash(C \cup G(D))) \cap(C \cup D)=\emptyset .
$$

We are now ready to present a new characterization of safety for the class of hybrid systems considered in this paper.

Theorem 6.1. Consider a hybrid system $\mathcal{H}=(C, F, D, G)$ such that (39) holds. Suppose that $\mathcal{H}^{-}=\left(C,-F, G(D), G_{D}^{-}\right)$satisfies (SA) and $X \subset \mathbb{R}^{n}$ is such that $\left(M 1_{X}\right)-\left(M 7_{X}\right)$ hold and $\tilde{C} \subset X$ with $\tilde{C}$ as in (17). Then, the hybrid system $\mathcal{H}$ is safe with respect to $\left(X_{o}, X_{u}\right)$, with $X_{o}$ closed, if and only if there exists a barrier function $B: \mathbb{R}_{\geq 0} \times \mathbb{N} \times$ $(C \cup D) \rightarrow \mathbb{R}$ that is locally Lipschitz on $\mathbb{R}_{\geq 0} \times \mathbb{N} \times((C \cup D) \cap X)$, and the following hold:

$$
\begin{array}{ll}
B(t, j, x) \leq 0 & \forall(t, j, x) \in \mathbb{R}_{\geq 0} \times \mathbb{N} \times X_{o}, \\
B(t, j, x)>0 & \forall(t, j, x) \in \mathbb{R}_{\geq 0} \times \mathbb{N} \times\left(X_{u} \cap(C \cup D)\right), \\
B(t, j+1, \eta) \leq 0 & \forall \eta \in G(x) \quad \text { and } \\
& \forall(t, j, x) \in K \cap\left(\mathbb{R}_{\geq 0} \times \mathbb{N} \times D\right), \\
G(x) \subset C \cup D & \forall(t, j, x) \in\left(\mathbb{R}_{\geq 0} \times \mathbb{N} \times D\right) \cap K,
\end{array}
$$

where

$$
K:=\left\{(t, j, x) \in \mathbb{R}_{\geq 0} \times \mathbb{N} \times(C \cup D): B(t, j, x) \leq 0\right\},
$$

and, for all $j \in \mathbb{N}$,

$$
\begin{aligned}
\alpha_{j}+\left\langle\zeta_{j}, F(x)\right\rangle \leq 0 \quad & \forall\left[\alpha_{j} \zeta_{j}^{\top}\right]^{\top} \in \partial_{P} B_{j}(t, x), \\
& \forall(t, x) \in \mathbb{R}_{\geq 0} \times \operatorname{int}(C),
\end{aligned}
$$

where $(t, x) \mapsto B_{j}(t, x):=B(t, j, x)$ for all $(t, x) \in \mathbb{R}_{\geq 0} \times \operatorname{int}(C)$.

In the following example, we illustrate how the assumptions used in Theorem 6.1 can be verified on a concrete example.

EXAMPLE 13. [Bouncing ball] Let us reconsider the bouncing ball hybrid model introduced in Example 2. We already showed in Example 11 that the system $\mathcal{H}=(C, F, D, G)$ satisfies (SA) and $\left(\mathrm{M} 1_{X}\right)-\left(\mathrm{M} 7_{X}\right)$ with respect to $X=C \backslash\{0\}$. The same properties can be shown for $\mathcal{H}^{-}=\left(C,-F, G(D), G_{D}^{-}\right)$by exploiting the symmetry between $\mathcal{H}$ and $\mathcal{H}^{-}$. Indeed, note that $G(D)=-D$ and $G_{D}^{-} \equiv-G$. Hence, $\mathcal{H}^{-}=(C,-F,-D,-G)$. Furthermore, by using the change of coordinates $x_{e}:=\left[\begin{array}{ll}x_{1} & -x_{2}\end{array}\right]^{\top}$, we conclude that the system $\mathcal{H}^{-}$ in the new coordinates, denoted $\mathcal{H}_{e}$, satisfies $\mathcal{H}_{e}^{-}=(C, F, D, G)=$ $\mathcal{H}$. Next, in order to verify (39), we notice that $D \subset C$; hence, $D \backslash(C \cup G(D))=\emptyset$. Finally, in order to conclude that $\tilde{C} \subset X$, we notice that $C$ is closed; hence, $\tilde{C} \subset C$. Furthermore, since $C=X \cup\{0\}$ and the solution starting from $\{0\}$ is trivial, we conclude that $\tilde{C} \subset X$.

\section{CONCLUSION}

In this paper, we proposed finite-horizon reachable sets for hybrid systems. Those reachable sets are viewed as set-valued maps for which we established the Lipschitz continuity property with respect to their arguments. The latter continuity property is shown to hold after restricting the data of the hybrid system to satisfy a set of conditions. Those conditions are made in order to handle the pathologies preventing such a regularity property from being always true. The usefulness of the latter study is illustrated when improving some of the existing converse safety statements in terms of barrier functions. As a future step, we propose to relax the proposed assumptions on the data in order to cover the case of hybrid systems with non-unique solutions.

\section{A APPENDIX}

LemmA A.1. Consider a differential inclusion $\mathcal{H}_{f}=\left(\mathbb{R}^{n}, F\right)$ which is pre-forward complete and such that $F$ is locally Lipschitz. Then, the set-valued maps $R^{b}$ and $R$ are locally Lipschitz.

Lemma A.2. Consider a constrained system $\mathcal{H}_{f}=(C, F)$ such that the following holds.

$(S A)_{f}$ The set $C$ is closed and $F$ is single valued and locally Lipschitz on $C$.

Condition $\left(M 3_{X}\right)$ is satisfied if, for any initial condition $x_{o} \in \partial C \cap X$ such that $\mathcal{S}\left(x_{o}\right)$ is nontrivial, either $F\left(x_{o}\right)=0$ or

$$
-F\left(x_{o}\right) \notin T_{C}\left(x_{o}\right) \text {. }
$$

Moreover, when $C$ is regular, (46) can be relaxed to

$$
F\left(x_{o}\right) \notin T_{\partial C}\left(x_{o}\right) \text {. }
$$

Lemma A.3. Consider a constrained system $\mathcal{H}_{f}=(C, F)$ such that $(S A)_{f}$ holds. Condition $\left(M 4_{X}\right)$ is satisfied if, for each $x_{o} \in \partial C \cap X$ such that $\mathcal{S}\left(x_{o}\right)$ is nontrivial,

$$
F\left(y_{o}\right) \in D_{C}\left(y_{o}\right) \quad \forall y_{o} \in U\left(x_{o}\right) \cap \partial C .
$$

Moreover, if the set $C$ is regular, condition (48) can be relaxed to

$$
F\left(x_{o}\right) \in D_{C}\left(x_{o}\right) \text {. }
$$




\section{REFERENCES}

[1] P. R. Wolenski and Y. Zhuang. Proximal analysis and the minimal time function SIAM journal on control and optimization, 36(3):1048-1072, 1998.

[2] B. Jakubczyk. Feedback linearization of discrete-time systems. Systems \& Control Letters, 9(5):411-416, 1987.

[3] D. Q. Mayne, J. B. Rawlings, C. V. Rao, and P.O Scokaert. Constrained model predictive control: Stability and optimality. Automatica, 36(6):789-814, 2000

[4] J. P. Aubin and A. Cellina. Differential Inclusions: Set-Valued Maps and Viability Theory, volume 264. Springer Science \& Business Media, 2012.

[5] J. P. Aubin. Viability Theory. Birkhauser Boston Inc., Cambridge, MA, USA, 1991

[6] R. P. Wolenski. The exponential formula for the reachable set of a lipschitz differential inclusion. SIAM Journal on Control and Optimization, 28(5):1148$1161,1990$.

[7] A. Dontchev and F. Lempio. Difference methods for differential inclusions: a survey. SIAM review, 34(2):263-294, 1992.

[8] M. Maghenem, B. Altin, and R. G. Sanfelice. Properties for reachability maps in well-posed hybrid systems. To appear in Proceedings of the 2020 IEEE American Control Conference (ACC), 2020.

[9] M. Maghenem and R. G. Sanfelice. Characterization of safety and conditional invariance for nonlinear systems. In Proceedings of the 2019 American Control Conference (ACC), pages 5039-5044, July 2019.

[10] S. Prajna and A. Rantzer. On the necessity of barrier certificates. IFAC Proceedings Volumes, 38(1):526-531, 2005.

[11] R. Wisniewski and C. Sloth. Converse barrier certificate theorems. IEEE Transactions on Automatic Control, 61(5):1356-1361, 2016.

[12] R. Goebel, R. G. Sanfelice, and A. R. Teel. Hybrid Dynamical Systems: modeling, stability, and robustness. Princeton University Press, 2012

[13] C. Cai, R. Goebel, R. G. Sanfelice, and A. R. Teel. Hybrid systems: limit sets and zero dynamics with a view toward output regulation. In Analysis and Design of Nonlinear control systems, pages 241-261. Springer, 2008.

[14] H. Frankowska. Local controllability of control systems with feedback. Fournal of Optimization Theory and Applications, 60(2):277-296, 1989.

[15] J. P. Aubin and H. Frankowska. Set-valued Analysis. Springer Science \& Business Media, 2009.

[16] F. H. Clarke, Y. S. Ledyaev, R. J. Stern, and P. R. Wolenski. Nonsmooth Analysis and Control Theory, volume 178. Springer Science \& Business Media, 2008.

[17] E. Michael. Continuous selections. I. Annals of mathematics, pages 361-382, 1956

[18] M. Maghenem and R. G. Sanfelice. Minimal-time functions in constrained nonlinear systems with applications to reachability analysis. To appear in Proceedings of the 2020 IEEE American Control Conference (ACC), 2020.

[19] M. Maghenem, A. Melis, and R. G. Sanfelice. Monotonicity along solutions to constrained differential inclusions. To appear in Proceedings of the 57th IEEE Conference on Decision and Control, 2020. Nice, France.

[20] M. Maghenem and R. G. Sanfelice. Characterizations of safety in hybrid inclusions via barrier functions. In Proceedings of the 22Nd ACM International Conference on Hybrid Systems: Computation and Control, HSCC '19, pages 109-118, NY, USA 2019. ACM 